Bundesgesundheitsbl - Gesundheitsforsch Gesundheitsschutz 2005 • 48:1061-1080 DOI 10.1007/s00103-005-1126-2

๑) Springer Medizin Verlag 2005

\title{
Infektionsprävention in Heimen
}

\section{Empfehlung der Kommission für Kranken- haushygiene und Infektionsprävention beim Robert Koch-Institut (RKI)}

nischen Versorgung aus Krankenhäusern in den Bereich der externen Betreuung ergeben sich auch außerhalb von Krankenhäusern dort Infektionsrisiken, die mit denen nosokomialer Infektionsrisiken in Krankenhäusern vergleichbar sein können. Die Einhaltung der zur Reduzierung derartiger Risiken erforderlichen Maßnahmen entsprechen der Forderung der WHO nach Lebensqualität im Alter und tragen auch zur Reduktion von Kosten bei.

Da die zur Infektionsprävention erforderlichen Maßnahmen weitgehend unabhängig vom Behandlungsort jedoch primär von der Art des medizinischen Eingriffs abhängig sind, ergeben sich zwangsläufig Überschneidungen zu anderen Empfehlungen dieser Richtlinie [2]. Bei weitgehender Übertragbarkeit der bewährten Maßnahmen wird deshalb in der jeweiligen Überschrift auf solche Empfehlungen verwiesen. Empfehlungen von grundlegender Wichtigkeit werden nochmals wiedergegeben, um hier eine für die tägliche Praxis selbstständig verwendbare Empfehlung zu geben. Abweichungen werden im Text deutlich gemacht.

\section{Geltungsbereich und Zielgruppe}

Diese Empfehlung gilt primär für solche Einrichtungen, in denen medizinische und damit assoziierte pflegerische Maßnahmen außerhalb von Krankenhäusern durchgeführt werden. Sie kann jedoch auch für andere Formen der Betreuung (z. B. Hauskrankenpflege) hilfreich sein.
Nicht behandelt werden in der vorliegenden Empfehlung Probleme und Anforderungen in speziellen Einrichtungen wie z. B. solche für geistig, psychisch oder anders schwer behinderte Personen sowie für Kinder. Diese Empfehlungen richten sich gleichermaßen an die Träger und die in den genannten Einrichtungen Tätigen.

\section{Infektionsrisiko}

Das Infektionsrisiko bei der Betreuung alter und pflegebedürftiger Menschen wird maßgeblich von der Abwehrsituation und den erforderlichen pflegerischen, medizinischen und hygienischen Maßnahmen bestimmt. Ursachen für ein erhöhtes Infektionsrisiko können z. B. chronische Erkrankungen (wie Diabetes mellitus), funktionelle Einschränkungen, Immobilität, Wunden (z. B. Decubitus) oder Bewusstseinstrübung (einschließlich Schluckstörung) sein.

Aber auch spezielle altersspezifische Aspekte müssen im Hinblick auf ein Infektionsrisiko im persönlichen Hygienemanagement hinreichend berücksichtigt werden. So ist beispielsweise eine strukturierte Mundhygiene mit regelmäßigem Zähneputzen und Prothesenpflege die Voraussetzung zur Karies-, Gingivitis- und Parodontitisprophylaxe mit Erhalt der physiologischen Mundhöhlenflora. Dadurch wird dem Risiko von Infektionen in der Mundhöhle einschließlich davon ausgehender weiterer Infektionen (z. B. Pneumonie, Endokarditis) entgegengewirkt [3]. 
Tabelle 1

Wichtigste Risikofaktoren für eine Besiedlung bzw. Infektion mit multiresistenten Erregern [16, 17, 18, 19, 38, 107, 125, $126,127]$

\section{Patienteneigene Faktoren \\ - Hohes Alter \\ - Immobilität}

- Funktionelle Störungen im Bereich der Nahrungsaufnahme

(z. B. Schluckstörung) oder der Ausscheidung

(z. B. Blasenentleerungsstörung)

- Multimorbidität, insbesondere chronische Erkrankungen

- Diabetes mellitus, Dialysepflichtigkeit

- Chronische Hautläsionen, Decubitalulcera, Ekzeme,

nässende Dermatitiden

\section{Externe Faktoren}

- Invasive Maßnahmen (Gefäßkatheter, Blasenkatheter, Ernährungssonden, Trachealkanülen)

-Wiederholte Antibiotikatherapien (insbesondere mit Chinolonen und 3. Generation-Cephalosporinen für das Auftreten von MRSA)

• Häufige Krankenhausaufenthalte
Die Anforderungen an die Infektionsprävention unterscheiden sich auch in Abhängigkeit von der Art der Betreuung, wobei sich im Rahmen einer allgemeinen $\mathrm{Zu}$ ordnung Risiken in erster Annäherung in solche bei „überwiegend sozialer Betreuung “ und solche bei „überwiegend pflegerischer Betreuung" (Behandlungspflege) einteilen lassen.

Gemeinsam ist den Einrichtungen, dass sich einige der dort lebenden Personen mehr oder weniger selbstständig versorgen können und im Allgemeinen mobil und nicht vorwiegend bettlägerig sind (überwiegend soziale Betreuung), während andere Bewohner eine dauernde, schwerwiegende individuelle Beeinträchtigung haben, die kontinuierliche Pflege und Behandlung erfordern (überwiegend pflegerische Betreuung, Behandlungspflege). Bei diesen Bewohnern sind Infektionen nicht selten und können sowohl endemisch als auch epidemisch als Ausbrüche, (s. auch • Tabelle 3) auftreten $[4,5,6,7,8,9,10,11,12,13]$. Deshalb ist gerade auch vor dem Hintergrund der persönlichen Hausarztwahl ein einheitlicher Standard insbesondere für die Maßnahmen erforderlich, die mit einem erhöhten Infektionsrisiko oder epidemischem Potenzial assoziiert sind.

Auch bei der ambulanten Betreuung pflegebedürftiger Menschen müssen Hygienestandards unter Berücksichtigung der individuellen Situation eingehalten werden. Dies erfordert ein hohes Maß an Flexibilität und Sachverstand. Die Unterbringung in Gemeinschaftseinrichtungen erhöht das Risiko von Infektionsübertragungen $[6,7,9]$. Akute Infektionen sind eine häufige Ursache für eine Kranken- hauseinweisung von Heimbewohnern. Sie sind auch am Tod von Bewohnern in Alten- und Pflegeheimen beteiligt [14, 15].

Ein weiteres zunehmendes Problem stellt die Besiedlung mit multiresistenten Erregern dar [7, 11, 12, 16, 17, 18, 19]. Ähnlich wie in Krankenhäusern treten in Alten- und Pflegeeinrichtungen, aber auch im Bereich der ambulanten Pflege, vermehrt multiresistente Bakterien auf, die durch Antibiotikaanwendung selektiert und z. B. bei Rückverlegung von Heimbewohnern aus medizinischen Einrichtungen in Heime hereingebracht werden können [20, 21, 22, 23, 24, 25]. Faktoren, die das Risiko für eine Kolonisation bzw. Infektion mit antibiotikaresistenten Erregern (z. B. MRSA, VRE, Enterobakterien) erhöhen, sind in $\bullet$ Tabelle 1 aufgelistet. Für Übertragungen innerhalb von Einrichtungen der Pflege kommen häufig die gleichen Wege in Betracht wie im Krankenhaus. Auch hier sind es in erster Linie die Hände des Personals.

Eine effektive Infektionsprävention setzt das geplante Zusammenspiel von Maßnahmen auf verschiedenen Ebenen der Betreuung voraus, deren Einzelaspekte im Folgenden dargestellt werden und als Grundlage für das Infektionspräventionskonzept einer Einrichtung dienen.

\section{Personelle und organisatorische Voraussetzungen}

Wesentliche Voraussetzungen zur Sicherstellung einer adäquaten Betreuung bzw. Pflege in Heimen werden im Heimgesetz (z. B. $\$ 11$ Abs. 1) formuliert [26]. Demnach ist es Aufgabe des Trägers
-... die Pflege nach dem allgemein anerkannten Stand medizinisch-pflegerischer Erkenntnisse sowie die ärztliche und gesundheitliche Betreuung zu sichern, - ... eine angemessene Lebensgestaltung zu ermöglichen,

- ... einen ausreichenden Schutz vor Infektionen zu gewährleisten und sicherzustellen, dass von den Beschäftigten die für ihren Aufgabenbereich einschlägigen Anforderungen der Hygiene eingehalten werden,

- ... zu gewährleisten, dass die persönliche und fachliche Eignung für die zu leistende Tätigkeit vorhanden ist, - ... ein Qualitätsmanagement betrieben wird.

Diese gesetzliche Verpflichtung stellt die Verantwortlichen vor die Aufgabe, durch Schaffung geeigneter personeller und organisatorischer Voraussetzungen sowie innerbetriebliche Regelungen zu gewährleisten, dass diese Anforderungen erfüllt sind. Je nach Art der Einrichtung wie auch der Pflegebedürftigkeit (siehe Kapitel 3) ergeben sich daraus unterschiedliche Anforderungen. Da alle Einrichtungen auf externe Dienste angewiesen sind (z. B. bewohnerbezogene ärztliche Betreuung mit freier Arztwahl, externe Dienstleister) ergibt sich die Notwendigkeit zu deren Koordination unter infektionspräventiven Aspekten (z. B. Kommunikation zwischen den betreuenden Ärzten).

Es gibt bisher keine umfassenden Untersuchungen über den Einfluss von Infektionspräventionsprogrammen und Infektionskontrollmaßnahmen auf das Auftreten von Infektionen bzw. deren Vermeidung in 
Heimen, die mit denen in Krankenhäusern vergleichbar wären $[27,28,29,30]$. Einige kontrollierte, randomisierte Studien belegen aber den Erfolg von Aus- und Weiterbildung des Personals hinsichtlich der Reduktion von Infektionen [31, 32]. Daher sind die folgenden Empfehlungen zu den personellen und organisatorischen Voraussetzungen der Infektionsprävention im Wesentlichen abgeleitet aus den umfangreichen $\mathrm{Er}$ fahrungen in Krankenhäusern, aber auch aus Ergebnissen einzelner Untersuchungen in Heimen und überzeugenden Hinweisen für ihre Wirksamkeit $[4,5,8,11,12,13,33]$.

\subsection{Fachpersonal (Alten- und Krankenpflegepersonal)}

Eine wesentliche Voraussetzung für eine adäquate Infektionsprävention ist grundsätzlich das Vorliegen der erforderlichen Sachkenntnis [34, 35, 36]. Dies betrifft alle relevanten Bereiche und erfordert je nach Größe und Pflegeanteil auch fest angestelltes Fachpersonal (siehe hierzu auch Heimpersonalverordnung mit der Vorgabe einer Fachkraftquote von 50\%) [34].

\subsection{Hygienebeauftragtes Personal}

Eine sachgerechte Umsetzung der hier gegebenen Empfehlungen ist in jeder Einrichtung am ehesten durch die Einsetzung eines Hygienebeauftragten mit entsprechender Fortbildung auf dem Gebiet der Infektionsprävention gewährleistet $[31,32]$. Zur Orientierung über Inhalte einer solchen Fortbildung kann die entsprechende Leitlinie der Deutschen Gesellschaft für Krankenhaushygiene (DGKH) dienen [37]. Die Aufgaben des Hygienebeauftragten werden zweckdienlich in einer Stellenbeschreibung festgelegt (QM/Heimgesetz).

\subsection{Hygienekommission}

Infektionsprävention kann nur als eine Aufgabe der gesamten Einrichtung erfolgreich sein. Insofern ist es erforderlich, für die Erörterung von Fragen der Infektionsprävention zwischen allen Beteiligten (Träger, Verwaltung, Ärzten und Pflegepersonal, evtl. Bewohner selbst oder deren Angehörige) ein geeignetes Forum zu schaffen, in dem einrichtungsspezifische Pro- blemlösungen erarbeitet werden. Zweckdienlich ist die Bildung einer regelmäßig tagenden „Hygienekommission“ - bestehend aus je einem Mitglied der relevanten Berufsgruppen ggf. unter Hinzuziehung eines beratenden Krankenhaushygienikers und evtl. unter Einbeziehung des gesetzlich vorgeschriebenen Heimbeirats. Aufgabe dieses Forums wäre die Festlegung der Schwerpunkte für die Infektionsprävention entsprechend den in der jeweiligen Institution gegebenen Erfordernissen und das Erreichen einer breiten Akzeptanz als Voraussetzung für den Erfolg.

\subsection{Kooperation mit niedergelassenen Ärzten}

Die Einbeziehung der persönlichen (vom jeweiligen Bewohner frei gewählten) Ärzte ist für eine effiziente und koordinierte Infektionsprävention von entscheidender Bedeutung. Die Koordinierung kann am ehesten durch Zusammenarbeit mit den Ärztekammern und den kassenärztlichen Vereinigungen erleichtert werden. Eine rechtzeitige, gezielte Diagnostik sowie eine individuell angepasste (erregerund infektionsgerechte) Therapie entscheiden darüber, ob Infektionen frühzeitig erkannt und wirksam behandelt werden können. Dies ist eine wesentliche Voraussetzung, um die Verbreitung von Krankheitserregern zu verhindern sowie Resistenzentwicklungen und der Selektion resistenter Bakterien vorzubeugen. Neben den individuellen mikrobiologischen Befunden und Therapieentscheidungen können unter Berücksichtigung fachspezifischer Leitlinien ggf. die Befunde anderer Mitbewohner hinsichtlich der epidemiologischen Beurteilung von Bedeutung sein (z. B. im Rahmen der Erkennung von Häufungen von Infektionen mit identischem Erreger). Deshalb ist eine kollegiale $\mathrm{Zu}$ sammenarbeit aller beteiligten Ärzte mit der Heimleitung, einer heiminternen Hygienekommission bzw. den Hygienebeauftragten unter Beachtung der ärztlichen Schweigepflicht, aber auch der gesetzlichen Vorgaben des Infektionsschutzgesetzes anzustreben. Grundlage einer derartigen Bemühung bildet die Verpflichtung eines jeden Arztes, die optimale Qualität seiner Arbeit zu gewährleisten. Bei pflegebedürftigen Heimbewohnern hat der be- treuende Arzt durch entsprechende Dokumentation sicherzustellen, dass seine ärztlichen Anordnungen (z. B. Art und Dauer der antibiotischen Therapie) vom Pflegepersonal korrekt umgesetzt werden können. Deshalb ist neben dem Hygieneplan auf Seiten der Ärzte auch der kontrollierte Umgang mit Antibiotika entscheidend: Selektion und Resistenzinduktion bei Bakterien sind wesentliche Voraussetzungen zur Entstehung von Infektionsproblemen mit multiresistenten oder so genannten „Hospital-Keimen“ $[18,24,38,39]$.

Als Ergebnis könnte sich ein „Qualitätszirkel Infektionsprävention “ etablieren, der alle betreuenden Ärzte unter Bezug auf die Verantwortung zur Qualitätssicherung zur Teilnahme motiviert, wofür die zuständigen Landesärztekammern Fortbildungspunkte gewähren, wenn die jeweiligen qualitativen Voraussetzungen gegeben sind.

\subsection{Hygieneplan und Infektionspräventionskonzept}

Gemäß $\$ 36$ Infektionsschutzgesetz (IfSG) [40] und der TRBA 250 [41] sind Einrichtungen nach $\$ 1$ Abs. 1, 1a des Heimgesetzes verpflichtet, innerbetriebliche Verfahrensweisen zur Infektionshygiene in Form von Hygieneplänen schriftlich festzulegen, mit dem Ziel, Infektionsrisiken für Bewohner und Personal in den betreffenden Einrichtungen zu minimieren. Im Kommentar zum IfSG [42] wird erläutert, welche Aspekte bei der Erstellung eines solchen Hygieneplans zu berücksichtigen sind:

- Analyse der einrichtungsspezifischen Infektionsgefahren (Risikoanalyse) in den verschiedenen Bereichen,

- Bewertung dahingehend, bei welchen Risiken risikominimierende Maßnahmen erforderlich sind,

- Festlegung konkreter Maßnahmen zur Risikominimierung,

- Methoden zur Überwachung der Einhaltung der Risikominimierungsmaßnahmen mit einem vertretbaren Aufwand,

- Festlegung von Zeitabschnitten, nach denen die Effizienz und Aktualität des Hygieneplans überprüft werden,

- Festlegung von Einzelheiten der Dokumentation des Hygieneplans und Schulung der Beteiligten. 
Der Hygieneplan sollte durch ein in unterschiedlichen Bereichen der Einrichtung tätiges Team (Hygienekommission) ggf. unter Einbeziehung des örtlichen Gesundheitsamts erstellt werden, wobei auf schon bestehende und bewährte Hygienepläne zurückgegriffen werden kann. Dabei wurde vom Gesetzgeber erkannt, dass „aufgrund der Unterschiedlichkeit der betroffenen Einrichtungen (...) an die Hygienepläne unterschiedliche Anforderungen zu stellen sind“ [42]. Eine beispielhafte Aufstellung von Themen und Inhalten eines Hygieneplans befindet sich im Anhang 1.

Das Heimgesetz ( $\$ 11$, Abs. 1 Nr. 9) fordert, einen ,ausreichenden Schutz der Bewohnerinnen und Bewohner vor Infektionen zu gewährleisten und sicherzustellen, dass von den Beschäftigten die für ihren Aufgabenbereich einschlägigen Anforderungen der Hygiene eingehalten werden “ [26].

Ein die Einrichtung umfassendes Infektionspräventionskonzept ist auch Teil des Qualitätsmanagements. Im $\$ 135$ a des SGB V [43] wird zum Qualitätsmanagement ausgeführt:

1. Die Leistungserbringer sind zur Sicherung und Weiterentwicklung der Qualität der von ihnen erbrachten Leistungen verpflichtet. Die Leistungen müssen dem jeweiligen Stand der wissenschaftlichen Erkenntnisse entsprechen und in der fachlich gebotenen Qualität erbracht werden.

2. Vertragsärzte, medizinische Versorgungszentren, zugelassene Krankenhäuser, Erbringer von Vorsorgeleistungen oder Rehabilitationsmaßnahmen und Einrichtungen, mit denen ein Versorgungsvertrag nach $₫ 111$ a besteht, sind nach $136 \mathrm{a}$, 136b, 137 und $137 \mathrm{~d}$ verpflichtet,

- sich an einrichtungsübergreifenden Maßnahmen der Qualitätssicherung zu beteiligen, die insbesondere zum Ziel haben, die Ergebnisqualität zu verbessern und

- einrichtungsintern ein Qualitätsmanagement einzuführen und weiterzuentwickeln.

Dies kann dadurch sichergestellt werden, dass Alten- und Pflegeheime

- über ausgebildetes Fachpersonal verfügen (Kat. IV, [34]),
- Hygienepersonal beschäftigen

(Kat. III),

- geeignete Maßnahmen zur Infektionsprävention festlegen (Hygieneplan)

(Kat. IV [42]),

- eine „Hygienekommission“/ggf. „Qualitätszirkel Infektionsprävention“ einrichten (Kat. III).

\section{Grundlegende Hygienemaßnahmen}

Für die verschiedenen Anliegen der Pflege sind Pflegestandards auf der Basis der Empfehlungen der einschlägigen Fachgesellschaften zu erstellen. Dabei müssen die Belange der Hygiene bei allen Abläufen hinreichend berücksichtigt werden.

\subsection{Händehygiene}

\section{(siehe hierzu auch die Empfehlung "Händehygiene" der Kommission für Krankenhaushygiene und Infektionsprävention [2])}

Die führende Rolle der Hände des Personals bei der Übertragung von Infektionserregern ist unbestritten. Demzufolge gilt die Händehygiene übereinstimmend als die entscheidende Maßnahme der Infektionsprävention. Im Rahmen des heiminternen Qualitätsmanagements ist sicherzustellen, dass bei allen pflegerischen Maßnahmen Möglichkeiten zur hygienischen Händedesinfektion gegeben sind. Für die Händehygiene gelten grundsätzlich die gleichen Anforderungen wie im Krankenhaus.

Die „hygienische Händedesinfektion“ ist insbesondere in folgenden Situationen erforderlich (alle Kat. IB):

- vor Tätigkeiten, die aseptisches Arbeiten erfordern (z. B. Bereitstellung von Infusionen, Zubereitung von Medikamenten),

- vor invasiven Maßnahmen, auch wenn dabei Handschuhe, ob steril oder unsteril, getragen werden (z. B. Anlage von Blasenkatheter, Punktion),

- vor Kontakt mit Bewohnern, die im besonderen Maße infektionsgefährdet sind (z. B. Immunsupprimierte),

- vor und nach Kontakt mit Körperbereichen, die vor Kontamination geschützt werden müssen (z. B. Wunden beim Verbandswechsel, Manipula- tionen an Venen-/Blasenkatheter, Tracheostoma, Infusionsbesteck),

- nach Kontakt mit Blut, Exkreten oder Sekreten, z. B. Drainageflüssigkeit,

- nach Kontakt mit infizierten oder kolonisierten Bewohnern, von denen Infektionen ausgehen können oder die mit Erregern von besonderer hygienischer Bedeutung besiedelt sind (z. B. MRSA),

- nach Kontakt mit potenziell kontaminierten Gegenständen, Flüssigkeiten oder Flächen (z. B. Urinsammelsysteme, Absauggeräte, Trachealtuben, Drainagen, Schmutzwäsche) und nach Ablegen von Einmalhandschuhen bei tatsächlichem oder möglichem Erregerkontakt oder nach sichtbarer Verunreinigung.

\subsection{Schutzkleidung}

Schutzkleidung im Sinne der TRBA 250 [41] ist jede Kleidung, die dazu bestimmt ist, Beschäftigte vor schädigenden Einwirkungen bei der Arbeit oder deren Arbeits- oder Privatkleidung vor der Kontamination durch biologische Arbeitsstoffe zu schützen und muss dem Personal zur Verfügung gestellt werden [42]. Durch Schutzkleidung (z. B. Überkittel, Handschuhe und Mund-Nasen-Schutz) soll außerdem eine Weiterverbreitung von Krankheitserregern vermieden werden [44, 45].

Die Auswahl der Schutzkleidung richtet sich nach

— der Art der pflegerischen/ärztlichen Tätigkeit und dem damit verbundenen Kontaminationsrisiko,

— der Pathogenität (ggf. auch Resistenz ) eines Keimes und dessen Übertragungsweges.

Daraus ergeben sich für die Schutzkleidung folgende Empfehlungen:

- Mund-Nasen-Schutz anlegen, wenn mit einer Exposition gegenüber infektiösen Aerosolen zu rechnen ist (z. B. Absaugen tracheostomierter Bewohner) (Kat. IV [41]),

- Handschuhe anlegen, wenn eine Exposition gegenüber Blut, Sekreten oder Exkreten möglich ist (z. B. Verbands- 
wechsel, Umgang mit Urindrainagesystemen) (Kat. IV [41]),

- Schürzen anlegen, wenn eine Kontamination der Berufskleidung der Körpervorderseite durch Blut, Sekrete oder Exkrete wahrscheinlich ist (z. B. Umgang mit Urindrainagesystemen, Wundversorgung) (Kat. IV [41]),

- Schutzkittel (langer Arm mit Bündchen), wenn mit Kontamination der Arme und der Kleidung durch Krankheitserreger zu rechnen ist (z. B. Pflegemaßnahmen bei Bewohnern mit Diarrhö, Versorgung größerer infizierter Wunden oder resistenter Keime) (Kat. IV [41]).

In diesen Fällen ist die Schutzkleidung bewohnerbezogen zu verwenden.

\subsection{Aufbereitung von Medizin- produkten und Pflegeartikeln}

\subsubsection{Medizinprodukte (MP) (siehe hierzu auch die Empfehlung „Anforderungen an die Hygiene bei der Aufbereitung von Medizin- produkten" der Kommission für Krankenhaushygiene und Infektionsprävention [2])}

Gemäß Medizinproduktegesetz (MPG) [46] und Medizinprodukte-Betreiberverordnung [47] ist die Aufbereitung von bestimmungsgemäß keimarm oder steril zur Anwendung kommenden Medizinprodukten unter Berücksichtigung der Angaben des Herstellers mit geeigneten validierten Verfahren so durchzuführen, dass der Erfolg dieser Verfahren nachvollziehbar gewährleistet ist und die Sicherheit und Gesundheit von Patienten, Anwendern oder Dritten nicht gefährdet wird (Kat. IV [46, 47]). Aus diesem Grund ist für diese Medizinprodukte eine detaillierte Aufbereitungsvorschrift mit Festlegung von Verantwortlichkeit und Verfahrenskontrollen zu erarbeiten.

\subsubsection{Pflegeartikel}

Je nach Einrichtung und Bewohner können bestimmte, insbesondere nicht personengebundene Pflegeartikel (z. B. Pediküre-, Maniküreset, Rasierapparat), zur Verbreitung von Krankheitserregern beitragen. Deshalb sind für die Maniküre und Pediküre bei jedem Bewohner ein eigenes
Set zu verwenden oder geeignete Maßnahmen der Desinfektion zu ergreifen. Wird die Hand- und Fußpflege von externen Dienstleistern durchgeführt, müssen für jeden Bewohner sachgerecht aufbereitete Instrumente verwendet werden. Auch elektrische Rasierapparate sollten bewohnerbezogen verwendet werden, andernfalls sind sie zwischen den Anwendungen hygienisch aufzubereiten.

Nachfolgend werden zu ausgewählten Pflegeartikeln Empfehlungen formuliert. Zusätzlich sind die entsprechenden Hygieneverordnungen der Bundesländer zu beachten (Kat. IV).

\subsubsection{Pflegegeschirr (Steckbecken/Urinflaschen etc.)}

Für die Aufbereitung von Pflegegeschirr sind vor allem aus Gründen der Verfahrenssicherheit (validiertes und in regelmäßigen Abständen kontrolliertes Verfahren), der Arbeitserleichterung und des Personalschutzes Reinigungs-DesinfektionsGeräte (RDG) anstelle manueller Aufbereitung zu bevorzugen (Kat. II).

\subsubsection{Waschschüsseln, Sitz-, Dusch- und Badewannen (siehe hierzu auch die Empfehlung „Anforderungen an die Hygiene bei der Reinigung und Desinfektion von Flächen" der Kommission für Krankenhaushygiene und Infektionsprävention [2])}

Bei Bewohnern mit bekannten Infektionen bzw. einer Kolonisation mit Erregern mit speziellen Resistenzen oder Multiresistenzen mit Übertragungsrisiko (z. B. Durchfall, multiresistente Erreger) sind Badewannen oder Waschschüsseln, insbesondere bei nachfolgender Benutzung durch andere Bewohner, desinfizierend zu reinigen (Kat. IB). Zusätzlich sollte bei Bewohnern mit erhöhtem Infektionsrisiko (z. B. nicht intakter Haut, Decubitus) sowohl vor als auch nach Benutzung eine desinfizierende Reinigung der Badewannen/Waschschüsseln durchgeführt werden (Kat. II).

\subsubsection{Betten- und Wäscheaufbereitung}

Obwohl in einigen Berichten auch Bettwäsche und der Umgang mit dieser als eine mögliche Quelle für die Verbreitung von Infektionserregern in Krankenhäu- sern angesehen wird [48, 49], liegen für den Bereich der Heime diesbezüglich keine Erfahrungen vor. Dennoch erscheint es sinnvoll, bei Vorliegen bestimmter Risikofaktoren (s. auch $\bullet$ Tabelle 2) eine desinfizierende Aufbereitung der Betten vorzunehmen. Die Aufbereitung von Matratzen wird erheblich erleichtert durch die Verwendung eines Schutzbezuges, der atmungsaktiv und desinfektionsmittelbeständig ist.

Da die Bettwäsche in der Regel nicht bewohnerbezogen verwendet wird und eine Mischung der Wäsche während des Aufbereitungsprozesses stattfindet, muss ein desinfizierendes Verfahren gewählt werden (z. B. Kochwäsche oder Waschen bei $60^{\circ} \mathrm{C}$ und Verwendung eines desinfizierenden Waschmittels). Bewohnereigene Wäsche (z. B. Kleidung) kann in der Regel wie Wäsche im Privathaushalt gewaschen werden. Während eines Ausbruchs von Erkrankungen mit Erregern, die durch Kontakt übertragen werden, sowie bei Personen mit bekannter MRSA-Kolonisation wird empfohlen, Leibwäsche, Handtücher und Waschlappen wie Bettwäsche der betroffenen Bewohner desinfizierend zu waschen.

Die Entsorgung der Wäsche erfolgt unmittelbar am Bett in geeignete Wäschesäcke, die bis zur endgültigen Entsorgung an einem dafür vorgesehenen Ort zwischengelagert werden können [41, 49a].

\subsection{Flächenreinigung und Flächendesinfektion}

(siehe hierzu auch die Empfehlung "Anforderungen an die Hygiene bei der Reinigung und Desinfektion von Flächen" der Kommission für Krankenhaushygiene und Infektionsprävention [2])

Unter infektionspräventiven Gesichtspunkten müssen Flächen sauber und trocken sein. Reinigungsutensilien (z. B. Reinigungstücher, Wischmobs) müssen in ausreichender Zahl vorhanden sein, maschinell-thermisch aufbereitet und vollständig getrocknet werden, um eine Vermehrung und Verschleppung von Mikroorganismen zu vermeiden. Eine Flächendesinfektion kann in Einrichtungen der Altenpflege in besonderen Situationen angezeigt sein. Die Beseitigung grober Kon- 


\section{Empfehlung}

Tabelle 2

Aufbereitung von Betten in Heimen

\begin{tabular}{|c|c|c|c|c|}
\hline & Gestell & Matratze & Bettzeug & Bettwäsche \\
\hline Routineverfahren & $\begin{array}{l}\text { Reinigung in regelmäßigen } \\
\text { Intervallen und bei Ver- } \\
\text { schmutzung }\end{array}$ & $\begin{array}{l}\text { Aufbereitung in der Regel } \\
\text { nicht erforderlich } \\
\text { Reinigung bei Verschmutzung }\end{array}$ & $\begin{array}{l}\text { Aufbereitung in der Regel } \\
\text { nicht erforderlich } \\
\text { Reinigung bei Verschmutzung }\end{array}$ & s. Punkt 5.3.3 \\
\hline $\begin{array}{l}\text { Kontamination mit } \\
\text { infektiösem Material }\end{array}$ & Wischdesinfektion & $\begin{array}{l}\text { Desinfektion, bei Verwendung } \\
\text { eines desinfektionsmittel- } \\
\text { beständigen Schonbezuges } \\
\text { Wischdesinfektion }\end{array}$ & $\begin{array}{l}\text { Desinfektion (thermisch oder } \\
\text { chemothermisch) }\end{array}$ & s. Punkt 5.3.3 \\
\hline $\begin{array}{l}\text { Wechsel des Nutzers/ } \\
\text { Bewohners ohne besonderes } \\
\text { Risiko }\end{array}$ & Reinigung & Reinigung & Reinigung & s. Punkt 5.3.3 \\
\hline $\begin{array}{l}\text { Wechsel des Nutzers/ } \\
\text { Bewohners mit besonderem } \\
\text { Risiko }^{\mathrm{a}}\end{array}$ & Wischdesinfektion & $\begin{array}{l}\text { Desinfektion, bei Verwendung } \\
\text { eines desinfektionsmittel- } \\
\text { beständigen Schonbezuges } \\
\text { Wischdesinfektion }\end{array}$ & $\begin{array}{l}\text { Desinfektion (thermisch oder } \\
\text { chemothermisch) }\end{array}$ & s. Punkt 5.3.3 \\
\hline
\end{tabular}

a Bewohner mit bekannter Infektionserkrankung oder bekannter Kolonisation mit multiresistentem Erreger.

taminationen mit potenziell infektiösem Material soll beispielsweise immer unter Anwendung einer gezielten Desinfektion erfolgen. Details müssen im Hygieneplan unter Berücksichtigung der individuellen Wohnverhältnisse (z. B. privates Einzelzimmer) geregelt werden.

In Einrichtungen oder Bereichen, in denen überwiegend soziale Betreuung erfolgt, ist in der Regel eine routinemäßige Reinigung wie im Haushalt ausreichend. Gezielte Desinfektionsmaßnahmen können nach Kontaminationen mit potenziell infektiösem Material in Einzelfällen (z. B. bei Gefahr einer Infektionsübertragung) angebracht sein.

\section{Gezielte Maßnahmen zur Infektionsvermeidung}

\subsection{Prävention von Harnwegsinfektionen}

\section{(siehe hierzu auch die Empfehlung „Prävention und Kontrolle Katheter-assoziierter Harnwegs- infektionen" der Kommission für Krankenhaushygiene und Infektionsprävention [2])}

Infektionen der Harnwege sind in Altenund Pflegeeinrichtungen die häufigsten Infektionen, überwiegend bedingt durch den Einsatz transurethraler Katheter. Zwischen $5 \%$ und $10 \%$ der in Pflegeheimen versorgten Personen sind katheterisiert; in Einrichtungen mit besonders pflegebedürftigen Personen sind die Raten noch höher und können bis 50\% betragen [11, 12 , 13]. Harnableitungen müssen wegen dieses Infektionsrisikos auf das unvermeidliche Minimum reduziert bleiben. Dies bedeutet, dass allein medizinische Indikationen die Voraussetzung für das Legen eines Blasenkatheters sein dürfen und diese vom behandelnden Arzt gestellt wird. Unter infektionspräventiven Aspekten sollten bei länger dauernder oder bleibender Inkontinenz Alternativen zu Verweilkathetern für die Harnableitung eingesetzt werden. Dies sind z. B. Kondom-Urinale, aufsaugende Medien (Vorlagen, Windeln) oder, bei entsprechender Indikation, operative Harnableitungen.

Auch bei nicht katheterisierten Bewohnern kann es, bedingt durch funktionelle Störungen oder chronische Krankheiten, zur Bakteriurie und damit potenziell zu Harnwegsinfektionen kommen.

Die Erreger von Harnwegsinfektionen stammen in der Regel aus der körpereigenen Keimflora der Bewohner selbst. Aber auch Übertragungen von einem Bewohner zu anderen, z. B. über Hände bzw. Gegenstände wie Urinflaschen, Steckbecken, Sammelgefäße und Urometer, sind möglich und können auch Ursache von Ausbrüchen sein $[50,51,52,53,54]$.

Für alle Formen der Harnwegskatheterisierung gilt deshalb (alle Kat. IB):
- Blasenkatheter dürfen nur nach strenger ärztlicher Indikationsstellung unter aseptischen Bedingungen angewendet werden und sind so bald wie möglich zu entfernen. Die durchführenden Personen müssen mit der Technik und den Erfordernissen der Aseptik und Antiseptik vertraut sein.

- Manipulationen am geschlossenen Harndrainagesystem (z. B. Urinabnahme) erfordern streng aseptische Handhabung und sollen auf das notwendige Minimum reduziert werden.

- Vor und nach jeder Manipulation am Blasenverweilkatheter oder Harndrainagesystem ist eine hygienische Händedesinfektion erforderlich.

- Spülungen und Instillationen sind nur bei spezieller urologischer Indikation, aber nicht zur Infektionsprophylaxe durchzuführen. Spülungen mit Antiseptika oder Antibiotika sind obsolet.

- Bei länger währender Drainage (>5 Tage) sollten unter Beachtung der Kontraindikationen suprapubische Blasenverweilkatheter bevorzugt werden.

\subsubsection{Harndrainage durch Katheterisierung der Harnblase 6.1.1.1 Transurethrale Katheter}

Blasenkatheter sind so bald wie möglich $\mathrm{zu}$ entfernen, um eine Harnröhrenstriktur und andere Komplikationen (wie Orchitis, Epididymitis, Prostatitis, Urethritis und Balanoposthitis) zu vermeiden. 
- Die Anlage des Katheters muss unter aseptischen Bedingungen von geschultem Personal möglichst mithilfe eines Katheterisierungssets durchgeführt werden (Kat. IB).

- Es dürfen nur sterile, geschlossene Ableitungssysteme eingesetzt werden (Kat. IA).

- Vor der sorgfältigen Vorbereitung aller benötigten Materialien müssen die Hände desinfiziert werden (Kat. IA).

- Die Schleimhautantiseptik erfolgt mit sterilen Tupfern und einem geeigneten Schleimhautantiseptikum z. B. auf Basis von PVP-Iod oder Octenidin. Nach Auflegen eines sterilen Schlitztuches wird steriles Gleitmittel instilliert und der Blasenkatheter vorsichtig unter sterilen Bedingungen (einschl. Pinzette, Handschuhe) eingeführt (Kat. IB).

- Das Blocken des Ballons erfolgt mit sterilem Aqua dest. oder vorzugsweise mit einer sterilen 8- bis 10\%igen Glycerin-Wasserlösung (nicht mit $\mathrm{NaCl}$ oder Leitungswasser) (Kat. IB).

- Danach erfolgen der Anschluss eines geschlossenen Drainagesystems mit Rückflussventil und die sichere Fixation (Kat. IB).

— Bei der täglichen Körperpflege erfolgt die Reinigung des Genitales mit Wasser und Seifenlotion ohne Zusatz antiseptischer Substanzen; dabei ist jeder Zug am Katheter zu vermeiden (Kat. IB).

\subsubsection{Suprapubische Katheter}

Die Anlage eines suprapubischen Katheters ist ein ärztlicher Eingriff. Ein Vorteil des suprapubischen Katheters liegt in der geringeren Keimbesiedelung der Bauchhaut im Vergleich zum Meatus urethrae. Zwar kommt es bei längerer Liegedauer auch hier zu einer Bakteriurie [55], jedoch ist das Keimspektrum in der Regel das der Hautflora und nicht, wie beim transurethralen Katheter, das der problembehafteten Perianalflora. Weitere Vorteile sind die einfache Pflege der Eintrittsstelle, die zuverlässige Vermeidung von Urethrastrikturen und subvesikalenulären Infektionen (wie Orchitis, Epididymitis u. a.) sowie die problemlose Kontrolle der Spontanmiktion und des Restharns.
- Eine tägliche Inspektion der Einstichstelle ist erforderlich, um eine Infektion an der Eintrittsstelle rechtzeitig zu erkennen. In der Regel kann die Punktionsstelle bei lange liegendem suprapubischen Katheter offen gelassen werden oder bedarf lediglich der Bedeckung durch ein kleines Pflaster (Kat. IB).

\subsubsection{Umgang mit Harndrainagen}

- Kein intermittierendes Abklemmen des Katheters (sog. Blasentraining) (Kat. IB). Um den Harnabfluss zu sichern, muss ein Abknicken von Katheter und Ableitungssystem vermieden werden.

- Der Auffangbeutel muss immer freihängend ohne Bodenkontakt unter Blasenniveau positioniert sein (Kat. IB).

- Der Katheter und der Drainageschlauch sollten nicht diskonnektiert werden (Kat. IA).

- Ist eine Diskonnektion nicht zu vermeiden (bzw. nach versehentlicher kurzzeitiger Diskonnektion), ist kein Wechsel von Katheter oder Drainagesystem erforderlich. Die erneute Verbindung von Katheter und Konus des Drainageschlauchs darf nur unter aseptischen Kautelen nach Wischdesinfektion mit einem alkoholischen Präparat wieder zusammengefügt werden (Kat. IB).

- Der Auffangbeutel ist rechtzeitig zu entleeren, bevor der Harn mit der Rückflusssperre in Kontakt kommt. Dazu sind Einmalhandschuhe zu tragen (Kat. IB).

- Das Auffanggefäß wird nach Gebrauch desinfizierend gereinigt (z. B. im Steckbeckenspülautomaten)

(Kat. III).

- Nach dem Ausziehen der Einmalhandschuhe ist eine Händedesinfektion erforderlich (Kat. IB).

- Ein Wechsel des geschlossenen Harndrainagesystems ist nicht routinemäßig in festen Intervallen erforderlich, sondern soll bei Bedarf nach individuellen Gesichtspunkten (z. B. bei Inkrustation, Obstruktion, Verstopfung, Katheter-induzierter symptomatischer Harnwegsinfektion) erfolgen (Kat. IB).

\subsubsection{Alternativen zu Verweilkathetern 6.1.3.1 Intermittierende Katheterisierung}

Beider Versorgung von Personen mit neurologischen Störungen hat sich die intermittierende Katheterisierung bewährt, da infektiologische Komplikationen reduziert werden können. Voraussetzung für die intermittierende Katheterisierung ist eine aseptische und atraumatische Technik sowie eine gute Patientencompliance $[55,56]$. Für die intermittierende Katheterisierung ist eine gute Schulung des Personals erforderlich. Nach adäquater Schulung können sich betroffene Personen auch selbst versorgen.

- Die intermittierende Katheterisierung erfordert die Schulung der Durchführenden hinsichtlich aseptischer und atraumatischer Techniken (Kat. IB).

\subsubsection{Kondom-Urinal}

Bei Inkontinenz männlicher Bewohner können ggf. Kondom-Urinale eingesetzt werden. Bei der Anwendung ist eine sorgfältige Pflege erforderlich, um lokale Komplikationen wie vor allem Hautmazerationen zu vermeiden. Dazu gehört eine sorgfältige genito-perineale Antiseptik, die Verwendung von transparenten Urinalen aus Silikon und das Abnehmen des Urinals über Nacht.

\subsubsection{Umgang mit Urostomata (lleum-/Colon-Conduit, operative Harnableitungen)}

Um eine optimale Stomaversorgung zu gewährleisten, sind vom gesamten Pflegepersonal wie auch vom Bewohner selbst die Grundsätze der Stomaversorgung einzuhalten $[57,58,59]$. Dies setzt eine ausführliche Information des Bewohners (und seines Lebenspartners) über den Versorgungswechsel nebst praktischer Anleitung voraus. In erster Linie ist der Bewohner selbst für sein Stoma verantwortlich. Andere Personen sollten nur in Ausnahmefällen oder bei genereller Pflegebedürftigkeit die Versorgung der Urostomie übernehmen.

Die Stomaversorgung ist umfangreich und komplex, hygienerelevante Einzelheiten umfassen u. a. folgende Aspekte:

- Der Beutel sollte bereits bei einer Füllung von etwa einem Drittel entleert 
werden, da er sonst zu schwer werden kann und nicht mehr sicher haftet.

- Grundsätzlich gilt, dass ein undichtes Versorgungssystem sofort gewechselt werden muss, unabhängig von der Liegezeit.

- Die Reinigung der peristomalen Haut wird mit warmem Wasser und keimarmem Tuch (z. B. Einmalkompressen oder einem frisch gewaschenen Waschlappen) durchgeführt, erforderlichenfalls unter Verwendung einer $\mathrm{pH}$-neutralen, parfümfreien Seife oder Waschlotion.

- Die Reinigung erfolgt dabei stets von innen nach außen, also vom Stoma weg.

- Um einer Hautirritation durch das Ausreißen einzelner Haare mit nachfolgender Follikulitis vorzubeugen, wird eine schonende Haarentfernung empfohlen.

\subsection{Prävention von Bakteriämie und Sepsis}

Eine sekundäre Bakteriämie, d. h. ausgehend von einem bestehenden Infektionsherd, ist die häufigste Ursache für septische Episoden. Etwa die Hälfte aller sekundären Bakteriämien wird durch Harnwegsinfektionen hervorgerufen; Hautinfektionen und Pneumonien machen je 10\%-15\% der Fälle aus [12]. In Anbetracht der relativen Häufigkeit von Gefäßkathetern bei schwer pflegebedürftigen Bewohnern besteht bei dieser Gruppe auch ein entsprechend hohes Risiko für Katheter-assoziierte Infektionen, die definitionsgemäß als primäre Bakteriämien bezeichnet werden.

\subsubsection{Punktionen und Injektionen (siehe hierzu auch die Empfehlung „Prävention Gefäßkatheter-assozi- ierter Infektionen" der Kommission für Krankenhaushygiene und Infek- tionsprävention [2])}

Vor jeder Punktion, z. B. Blutabnahme, und Injektion (subkutan, intramuskulär, intravenös) muss eine sorgfältige Hautantiseptik mit einem alkoholischen Präparat und sterilisiertem Tupfer durchgeführt werden. Durch konsequent aseptische Handhabung muss eine Kontamination des $\mathrm{Zu}-$ behörs sowie ggf. der Medikamentenlösungen ausgeschlossen werden [6o]. Aus Gründen des Personalschutzes ist das Tra- gen von Einmalhandschuhen erforderlich, wenn Blutkontakt möglich ist [41].

- Hygienische Händedesinfektion vor jeder Zubereitung von Injektionslösungen sowie vor jeder Punktion oder Injektion (Kat. IB),

- Desinfektion der Einstichstelle mit einem alkoholischen Präparat und sterilisierten Tupfer (Kat. IB),

- Tragen von Einmalhandschuhen bei möglichem Blutkontakt (Kat. IV [41]),

- Eindosisbehälter sollten bevorzugt vor Mehrdosisbehältern verwendet werden (Kat. IB). Insbesondere bei Verwendung von lipid- und proteinhaltigen Lösungen strikte Beachtung der Herstellerangaben (Kat. IV, [46]),

- Gummimembran des Mehrdosisbehälters mit einem geeigneten alkoholischen Desinfektionsmittel desinfizieren, Einwirkzeit beachten (s. auch Angaben des Arzneimittelherstellers) (Kat. IB),

- bei jeder Punktion des Mehrdosisbehälters müssen die Spritze und Kanüle gewechselt werden (Kat. IB),

- angebrochene Mehrdosisbehälter müssen mit Datum und Uhrzeit des Erstanbruchs versehen sein (Kat. IB),

- Zumischung von Arzneimitteln bzw. Herstellung von Arzneimittelmischungen erst unmittelbar vor Injektion,

- Standzeiten und Lagerungsbedingungen sind den Herstellerangaben zu entnehmen (Kat. IV [46]).

Bei s.c.-Insulininjektionen (mit oder ohne Pen), die der Bewohner selbst vornimmt, kann eine Hautdesinfektion unterbleiben; werden sie jedoch vom Personal vorgenommen, ist aus haftungsrechtlichen Gründen in jedem Fall eine vorherige Hautdesinfektion durchzuführen. Injektionsnadeln sind nach den Vorgaben des Herstellers zu verwenden und zu wechseln. Die für eine Mehrfachverwendung vorgesehenen Kanülen sind nur bei Anwendung durch den Bewohner selbst entsprechend zu handhaben. Personal benutzt jeweils eine neue Kanüle. Angebrochene, in Verwendung befindliche Insulinpens dürfen in der Regel nicht im Kühlschrank gelagert werden (Herstellerangaben beachten).
Bei nicht-bewohnerbezogener Anwendung sind ein Nadelwechsel nach jeder Injektion und eine vorherige Hautdesinfektion erforderlich.

- Bei bewohnerbezogener Anwendung

- täglicher Nadelwechsel,

- keine Hautdesinfektion erforderlich, wenn die Injektion durch den Bewohner selbst erfolgt,

- Lagerung der in Gebrauch befindlichen Pens nach Angaben des Herstellers (in der Regel bei Zimmertemperatur ausreichend), Lagerung der Vorräte nach Angaben des Herstellers (in der Regel im Kühlschrank).

\subsubsection{Intravasale Katheter und Infusionstherapie} (siehe hierzu auch die Empfehlung „Prävention Gefäßkatheter assoziierter Infektionen" der Kommission für Krankenhaushygiene und Infektionsprävention [2])

Bei der Anlage und Versorgung von intravasalen Kathetern müssen alle für die Versorgung von Patienten in Krankenhäusern etablierten Hygienemaßnahmen beachtet werden. Insbesondere sind Händedesinfektion, sorgfältige Hautantiseptik vor der Punktion und Non-touch-Technik beim Verbandswechsel wichtig.

Eine hygienische Händedesinfektion ist vor Kontakt mit aseptisch zu handhabenden Materialien, vor Konnektion/Diskonnektion des Infusionssystems, vor dem Zuspritzen von Arzneimitteln erforderlich (Kat. IB).

- Diskonnektionen sind auf ein absolutes Minimum zu beschränken (Kat. IB).

- Nach jeder Diskonnektion muss ein neuer, steriler Verschlusstopfen verwendet werden (Kat. IB).

- Venenverweilkanülen können so lange liegen bleiben, wie sie klinisch benötigt werden und keine Komplikationszeichen feststellbar sind (Kat. IB).

\subsection{Prävention von Atemwegsinfektionen}

Infektionen der Atemwege einschließlich der Virusgrippe sind die zweithäufigsten Infektionen bei älteren Menschen. 
Schwer pflegebedürftige Bewohner sind nicht nur durch altersbedingte physiologische Veränderungen der respiratorischen Funktionen, sondern auch durch disponierende Grundkrankheiten, Immobilität oder Bewusstseinstrübung in erheblichem Maß gefährdet, eine Pneumonie zu entwickeln $[4,6,12]$. Auf einen Zusammenhang zwischen Kontamination des Trinkwassersystems und endemischen und epidemischen Legionellosen bei älteren Personen sei hingewiesen [ 61 , 62, 63].

Besondere Beachtung verdient die Lungentuberkulose, da der Altersgipfel bei den Tuberkuloseerkrankungen in Deutschland z. B. 2002 oberhalb von 69 Jahren lag [64]. Bedingt durch die Beeinträchtigung insbesondere der zellulären Immunität im höheren Lebensalter gehören die meist älteren Bewohner von Pflegeheimen zu einer Population mit typischerweise erhöhtem Risiko für eine aktive Tuberkulose. Im Allgemeinen tritt bei Senioren die Tuberkulose in Form einer Reaktivierung einer früher akquirierten Tuberkulose auf [65]. Aber auch exogene Re-Infektionen sind möglich, da viele Menschen im Alter die in früheren Lebensjahren erworbene Tuberkulinreaktivität verlieren [66]. Daher haben in Deutschland Personen, die in ein Altenpflegeheim aufgenommen werden sollen, gemäß $\$ 36$ (4) IfSG ein ärztliches Zeugnis darüber vorzulegen, dass bei ihnen keine Anhaltspunkte für das Vorliegen einer ansteckungsfähigen Lungentuberkulose vorhanden sind. Dies schließt jedoch eine weitere differenzialdiagnostische Berücksichtigung bei Auftreten entsprechender Symptome nicht aus. Beim Auftreten neuer Tuberkuloseerkrankungen in einer Pflegeeinrichtung müssen notwendige Umgebungsuntersuchungen und Maßnahmen der Chemoprophylaxe bzw. -prävention in Absprache mit dem zuständigen $\mathrm{Ge}$ sundheitsamt erfolgen.

\subsubsection{Inhalation (siehe hierzu auch die Empfehlung „Prävention der nosokomialen Pneumonie" der Kommission für Krankenhaushygiene und Infektionsprävention [2])}

Bei der Inhalationstherapie muss konsequent aseptisch gearbeitet werden, $d . h$.
Händedesinfektion vor Manipulationen an den Geräten, vollständige Aufbereitung von Inhalationsgeräten und Verneblern nach jedem Gebrauch und ausschließliche Verwendung steriler Flüssigkeiten sind essenzielle Maßnahmen zur Prävention tiefer Atemwegsinfektionen [67]. Bei der Aufbereitung von Inhalationsgeräten müssen auch die Angaben des Herstellers berücksichtigt werden.

\subsubsection{Tracheostomapflege (siehe hierzu auch die Empfehlung „Prävention von postoperativen Wundinfektionen" der Kommission für Krankenhaushygiene und Infektionsprävention [2])}

Bei der Versorgung eines Tracheostomas muss zwischen frischen und alten Tracheotomien unterschieden werden. Bei Heimbewohnern, bei denen im Rahmen eines kürzlich zurückliegenden Krankenhausaufenthaltes ein Tracheostoma neu angelegt wurde und bei denen es noch nicht zur Abheilung der Wunde mit Ausbildung eines Stomakanals (2-4 Wochen nach Anlage) gekommen ist, stellt die Pflege des Tracheostomas dieselben Anforderungen wie eine frische Operationswunde [68]. Wichtig sind eine vorherige Händedesinfektion, das Tragen von Einmalhandschuhen und ein aseptisches Vorgehen (Nontouch-Technik).

Nach Abheilung der Tracheotomiewunde soll die Umgebung des Tracheostomas mehrmals täglich mit einem jeweils frischen Waschlappen, Mullkompressen bzw. handelsüblichen Reinigungstüchern feucht gereinigt werden. Evtl. vorhandene Krusten bzw. Borken werden mit einer Pinzette oder mit einem mit physiologischer Kochsalzlösung getränkten Tuch entfernt. Um eine Schädigung der Haut zu vermeiden, muss das Stoma sicher vor Feuchtigkeit und Schleim geschützt werden. Bei der Säuberung und Versorgung des Tracheostomas muss das Personal Einmalhandschuhe tragen. Für Bewohner, die sich selbst versorgen, ist Händewaschen ausreichend.

\subsubsection{Wechsel der Trachealkanüle}

Sofern es erforderlich ist, sollte der Bewohner zunächst abhusten oder ggf. abgesaugt werden (s. auch 6.3.4). Danach wird die Trachealkanüle mit Einmalhand- schuhen entfernt und z. B. in eine Nierenschale abgelegt. Bei einem frischen Tracheostoma $(<2-4$ Wochen nach Anlage) muss die neue sterile Kanüle unter aseptischen Bedingungen eingesetzt und fixiert werden.

Bei einem Langzeit-Tracheostoma mit Ausbildung eines Stomakanals müssen neu einzusetzende Trachealkanülen nicht steril sein, eine Desinfektion ist ausreichend. Durch vorsichtige Handhabung beim Neueinsetzen muss eine Kontamination der aufbereiteten Trachealkanüle durch unbeabsichtigten Kontakt mit potenziell kontaminierten Flächen oder Gegenständen vermieden werden. Bei der Versorgung solcher Bewohner muss sich das Personal nach Entfernung der alten Kanüle und vor Einsetzen der aufbereiteten Kanüle die Hände desinfizieren und Einmalhandschuhe tragen.

- Vor und nach Manipulationen am Tracheostoma bzw. an der Trachealkanüle erfolgt eine hygienische Händedesinfektion (Kat. IB).

- Bei frischem Tracheostoma muss beim Wechsel der Trachealkanüle eine sterile Kanüle unter aseptischen Bedingungen eingesetzt werden (Kat. IA).

- Bei Trachealkanülen-Wechsel bei Langzeit-Tracheostoma ist die Verwendung einer desinfizierten Trachealkanüle ausreichend (Kat. II).

- Aufbereitung der entfernten Kanüle gemäß Herstellerangaben (Kat. IB).

\subsubsection{Endotracheales Absaugen (siehe hierzu auch die Empfehlung „Prävention der nosokomialen Pneumonie" der Kommission für Krankenhaushygiene und Infektionsprävention [2])}

Das endotracheale Absaugen (z. B. frisch tracheotomierter Bewohner) muss unter aseptischen Bedingungen erfolgen. Um das aseptische Arbeiten zu erleichtern, sollte möglichst zu zweit gearbeitet werden. Vorsichtiges Vorgehen ist wichtig, um Kontaminationen und Schleimhautverletzungen zu vermeiden. Es soll nicht routinemäßig in festen Intervallen abgesaugt werden, sondern nur bei einer die Atmung behindernden Sekretansammlung. Da Verspritzen von respiratorischem Sekret mög- 
lich ist, wird das Tragen eines Mund-Nasen-Schutzes sowie einer Schutzbrille zum Schutz des Personals empfohlen.

- Endotracheale Absaugung unter aseptischen Bedingungen durchführen (Kat. IB).

- Hygienische Händedesinfektion vor und nach dem endotrachealen Absaugen (Kat. IA).

- Zum Absaugen sind keimarme Einmalhandschuhe zu tragen (Kat. IV [41]).

- Bei Anwendung des offenen Absaugverfahrens sind sterile Einmalkatheter zu verwenden (Kat. IB). Innerhalb eines Absaugvorgangs kann derselbe Katheter mehrfach verwendet werden; zur Spülung ist dabei steriles Wasser zu verwenden (Kat. IA).

\subsection{Prävention von Haut- und Weichteilinfektionen}

Druckgeschwüre. (Decubitalulcera) sind diehäufigste Ursache von Haut- und Weichteilinfektionen in Pflegeheimen und treten bei bis zu 20\% der dort versorgten Personen mit der Gefahr einer daraus resultierenden Osteomyelitis oder Sepsis auf $[6,13]$.

Neben den bekannten Risikofaktoren für eine Besiedlung mit Krankheitserregern spielen für die Entwicklung von Decubitalulcera Immobilität, Druck, Zug, Scherkräfte, Feuchtigkeit, Inkontinenz, Steroidtherapie, schlechter Ernährungszustand [4] sowie Exsikose eine wichtige Rolle. Chronische Ulcera können mit einer Vielzahl verschiedener Erreger besiedelt und über lange Zeit ein Reservoir für multiresistente $\mathrm{Er}$ reger, insbesondere MRSA, sein [11].

Bei Decubitus-gefährdeten Bewohnern muss alles getan werden, um einen Decubitus zu vermeiden, seine frühzeitige Entwicklung zu erkennen und seine Ausbildung im Anfangsstadium zu verhindern. Dazu gehören die Erstellung eigener Pflegestandards auf der Basis der Empfehlungen der einschlägigen Fachgesellschaften (s. auch nationaler Decubitusstandard [69]) und die rechtzeitige Verwendung von Spezialbetten/-matratzen zur Verhinderung von Decubitalulcera.

Krätzmilbe. Auf die Krätzmilbe als Infektionserreger der Scabies ist besonderes
Augenmerk zu richten. Die Scabies ist eine kontagiöse Hauterkrankung und kann auch in Alten- und Pflegeheimen zu Problemen führen. In den letzten Jahren wurde wiederholt über epidemische Verbreitungen von Scabies in Heimen mit einer hohen Befallsrate unter Bewohnern und Personal und langer Persistenz berichtet [4, 70, 71]. Ist beim Auftreten von juckenden Hautläsionen auch der begründete Verdacht auf eine Scabies gegeben, sind neben der Veranlassung einer fachärztlichen dermatologischen Untersuchung die notwendigen Hygienemaßnahmen einzuleiten. Hierzu wird auf die einschlägigen Merkblätter des RKI und der Gesundheitsbehörden der Länder verwiesen.

\subsubsection{Wundverbände \\ (z. B. Decubitus, Ulcus cruris) (siehe hierzu auch die Empfehlung „Prävention von postoperativen Wundinfektionen" der Kommission für Krankenhaushygiene und Infektionsprävention [2])}

Sekundär heilende und sezernierende Wunden benötigen eine sterile Wundauflage, um das Wundsekret aufzufangen und die Wundheilung zu unterstützen. Weil bei allen offenen Wunden eine exogene Kontamination möglich ist, müssen Verbandswechsel unter aseptischen Vorsichtsmaßnahmen mit der sog. Nontouch-Technik durchgeführt werden [68, 72]. Auch jede Spülflüssigkeit muss steril sein. Leitungswasser ist nicht frei von Mikroorganismen. Bei Verwendung von Sterilgütern muss das Verfallsdatum beachtet werden. Angebrochene Sterilverpackungen müssen am Ende des Verbandswechsels verworfen werden. Besiedelte oder infizierte Wunden können ein potenzielles Erregerreservoir darstellen und erfordern deshalb einen gut sitzenden, das Sekret aufnehmenden bakteriendichten Verband. Durch umsichtiges Arbeiten muss außerdem eine direkte oder indirekte Kontamination der Umgebung verhindert werden. In der Regel sollen deshalb aseptische Wunden vor septischen Wunden verbunden werden.

- Besteht das Risiko der Kontamination der Arbeitskleidung, wird bei großen Wundflächen eine Einmalschürze/Kittel getragen (Kat. IV [41]).
- Vor dem Verbandswechsel müssen die Hände desinfiziert und ggf. keimarme Einmalhandschuhe (z. B. bei durchfeuchtetem Verband) angezogen werden (Kat. IB).

- Nach Abnehmen des Wundverbandes (festklebende wundabdeckende Kompressen mit steriler Pinzette entfernen) werden die Einmalhandschuhe entsorgt (Kat. IB).

- Erneute Händedesinfektion (Kat. IB).

- Die Wundbehandlung erfolgt nach hygienischer Händedesinfektion in No-touch-Technik mit sterilen Instrumenten oder mit sterilen Handschuhen nach den Vorgaben der behandelnden Ärzte (Kat. IB).

- Zum Spülen von Wunden dürfen nur sterile Lösungen verwendet werden (Kat. IB).

- Hinsichtlich der Haltbarkeit der für die Spülungen verwendeten Lösungen müssen die Angaben des Herstellers beachtet werden (Kat. IV [46]).

- Benutztes Instrumentarium wird unmittelbar nach Gebrauch unter Vermeidung der Kontamination des Umfeldes sicher entsorgt (Kat. IV [41]).

\subsubsection{Stomata}

Bei der Versorgung von Stomata (Enterostoma, Urostoma) muss zwischen frischen und abgeheilten Stomawunden unterschieden werden. Maßnahmen an frischen Wunden erfolgen im Krankenhaus unter aseptischen Bedingungen, d. h. mit sterilen Instrumenten, sterilem Verbandmaterial und mit Non-touch-Technik. Nach Neuanlage eines Enterostomatas sollte der Patient vor Verlegung in ein Heim in die Stomapflege eingewiesen worden sein bzw. initial durch einen Stomatherapeuten weiter betreut werden.

Nach Abheilung der Stomawunde ist die sorgfältige Pflege der Haut um das Stoma zur Vermeidung von Hautirritationen und nachfolgender Infektion wichtig [73]. Zur Reinigung werden z. B. feuchte Kompressen mit einer $\mathrm{pH}$-neutralen Waschlösung verwendet. Ein selbstständiger Heimbewohner führt die Stomapflege nach Waschen der Hände selbst durch. Bei pflegebedürftigen Heimbewohnern erfolgt die Stomaversorgung durch das Pflegepersonal nach vorheriger Händedesinfektion und unter Tragen von Einmalhandschu- 
hen und ggf. flüssigkeitsdichter Schürze. Bei bettlägerigen Bewohnern muss das Bett mit einer Unterlage vor Kontamination z. B. mit Darminhalt geschützt werden.

- Bei der Stomapflege muss das Pflegepersonal Einmalhandschuhe tragen (Kat. IV [41]).

- Vor und nach Manipulationen an Stomata ist eine hygienische Händedesinfektion durchzuführen (Kat. IB).

\subsection{Prävention gastrointestinaler Infektionen}

Durchfälle kommen bei betagten Menschen häufig auch ohne infektiöse Ursache vor. Dennoch sind Durchfallerkrankungen oft auf Infektionen durch virale und bakterielle Erreger zurückzuführen [12, 74]. Ein hoher Anteil inkontinenter Bewohner begünstigt dabei Übertragungen [75]. Die altersbedingte Zunahme des Magensaft-pHWertes macht alte Menschen besonders anfällig für lebensmittelbedingte gastrointestinale Infektionen, die in Pflegeheimen auch zu Ausbrüchen führen können [4, 11, 12]. Deshalb ist die Beachtung notwendiger Hygienemaßnahmen im Umgang mit Nahrungsmitteln (Lagerung, Verarbeitung) sowie bei der Aufbewahrung von Speisen erforderlich (s. auch Kapitel 13).

\subsubsection{Sondenernährung}

Sondennahrung stellt für viele Mikroorganismen ein gutes Nährmedium dar. Daher ist eine hygienische Händedesinfektion vor Verabreichung der Nahrung bzw. vor Spülen der Sonden erforderlich $[76$, $77,78,79,80,81]$.

Generell sollte eine raumtemperierte Sondenkost verwendet werden, d. h., kommerziell erhältliche Sondennahrung sollte nicht im Kühlschrank, sondern bei Raumtemperatur gelagert werden. Im Einzelfall sind Abweichungen möglich. Wenn eine warme Sondennahrung gewünscht wird, kann diese - bei Glasflaschen - statt in einem Wasserbad besser langsam in der Mikrowelle bis max. $40^{\circ} \mathrm{C}$ erwärmt werden. Sie ist dann jedoch innerhalb von 4-5 Stunden zu applizieren. Angebrochene Sondenkostflaschen sollten im Kühlschrank gelagert und Reste (wegen der Gefahr zunehmender bakterieller Kontamination) spä- testens nach 24 Stunden verworfen werden. Bei pumpengesteuerter Applikation mit niedriger Laufrate kann kühlschrankkalte Sondennahrung verwendet werden, da diese sich zwischenzeitlich im Überleitsystem an die Raumtemperatur anpasst.

Zur intermittierenden Nahrungsgabe soll entweder eine neue oder eine sachgerecht aufbereitete Spritze (s. auch 5.3.1) verwendet werden, da verbliebene Nahrungsreste ein gutes Nährmedium für Mikroorganismen darstellen. Plastikbeutel mit angeschweißtem Überleitungssystem sollten spätestens nach 24 Stunden verworfen werden. Bei Personen, die bettlägerig sind und auf flüssige oder pürierte Kost und/ oder aufgrund geistiger oder funktioneller Einschränkungen bei der Nahrungsaufnahme auf fremde Hilfe angewiesen sind, muss durch entsprechende Verhaltensweisen bzw. pflegerische Maßnahmen eine (Mikro-)Aspiration von Nahrung verhindert werden. Ein Infektionsrisiko bei Aspiration kann dadurch vermindert werden, dass zum Verflüssigen pürierter Kost nur frisch abgekochtes Trinkwasser verwendet wird.

Pulverförmige Nahrung muss kontaminationsfrei in portionsgerechten Mengen zum sofortigen Verbrauch zubereitet und immer mit abgekochtem Wasser angerührt werden. Ebenso soll Wasser, das zur Zubereitung von Tee oder Instanttee dient, zuvor abgekocht werden. Schüttelbecher, Messlöffel etc. sind bei erneutem Gebrauch mit geeigneten Verfahren (z. B. Geschirrspülmaschinen bei Temperatur $>60^{\circ} \mathrm{C}$ oder in Reinigungs-Desinfektions-Geräten) thermisch desinfizierend aufzubereiten.

Nach jeder Nahrungsgabe muss die enterale oder die perkutane enterale Gastrostomie (PEG-)Sonde mit zuvor frisch zubereitetem (kochendes Wasser verwenden) und auf Körpertemperatur abgekühltem Tee oder abgekochtem und abgekühltem Wasser durchgespült werden, um eine Verstopfung der Sonde zu verhindern. Früchtetee und schwarzer Tee führen zur Ausflockung von Nahrungsresten und sollten daher nicht verwendet werden. Bei einer transnasalen Sonde ist die tägliche Pflege mit Reinigung von Naseneingang und Sonde mit Wasser und ggf. Seife empfehlenswert. Zur Pflege des Nasenflügels ist neben einer Nasensalbe die Fixierung der Sonde durch ein schonendes Pflaster wichtig [8o].
Wurde bei pflegebedürftigen oder noch selbstständigen Heimbewohnern eine PEG-Sonde neu angelegt, muss in den ersten 1 bis ca. 10 Tagen nach Anlage täglich ein steriler Verbandswechsel erfolgen. Vor Durchführung des Verbandswechsels ist eine hygienische Händedesinfektion erforderlich, es sollten Einmalhandschuhe getragen werden. Zur Vermeidung einer lokalen Wundinfektion muss für trockene und keimarme Wundverhältnisse gesorgt werden. Von zu intensiver Wundpflege mit täglicher Desinfektion der Einstichstelle oder gar Applikation von Salben ist abzuraten, um Mykosen und Ekzeme der Bauchhaut zu vermeiden. Die Pflege der äußeren Haut kann mit Wasser und milder Seife erfolgen. Die Sondeneintrittsstelle muss vollkommen trocken sein, bevor ein neuer Verband angelegt wird, da eine feuchte Kammer eine lokale Infektionsentstehung begünstigt.

Nach initialer Wundheilung, die meistens 7-10 Tage nach Neuanlage abgeschlossen ist, genügen Verbandswechsel in 2- bis 3-tägigen Abständen. Nach vollständiger Abheilung und Granulation des Stomakanals (etwa 2-4 Wochen nach Neuanlage) ist ein Verband nicht zwingend erforderlich, wenn sorgsame Pflege und kritischer Umgang mit dem Sondensystem gewährleistet sind. Grundsätzlich sind alle Artikel zur Applikation von Sondennahrung Einwegartikel und sollten nach 24 Stunden erneuert werden.

Ein routinemäßiger Sondenwechsel ist nicht erforderlich, eine sorgfältig gepflegte PEG-Sonde kann 2-5 Jahre in situ verbleiben. Es ist empfehlenswert, dass der betreuende Arzt die Sondeneintrittsstelle unmittelbar nach Anlage einer PEG-Sonde täglich, nach erfolgter Abheilung und Ausbildung eines Stomakanals anfangs alle 24 Wochen und später in längeren Intervallen hinsichtlich Hautveränderungen beurteilt. Bei Rötungen, Pustel- oder eitriger Sekretbildung, die dem Bewohner oder Pflegepersonal auffallen, oder bei lokalen Schmerzen ist dies dem behandelnden Arzt sofort mitzuteilen. Zu Materialien, Sondenpflege und Mehrfachverwendung von Applikationssystemen wird auf Pflegerichtlinien für die Langzeitbetreuung von Patienten mit enteralen Sondensystemen verwiesen [80]. 
- Bei Bewohnern, die über eine enterale Sonde oder PEG-Sonde ernährt werden, soll die Mundpflege mit frisch abgekochtem und abgekühltem Wasser oder frisch zubereitetem Tee (siehe oben) oder sterilem Aqua dest. durchgeführt werden (Kat. IB).

- Hygienische Händedesinfektion vor Verabreichung der Nahrung bzw. vor Spülen der Sonden (Kat. IB).

- Bei intermittierender Nahrungsgabe jeweils Verwendung entweder einer neuen oder hygienisch aufbereiteten Spritze (Kat. IB).

- Wechsel von Plastikbeutel mit angeschweißtem Überleitungssystem nach Herstellerangaben (Kat. IV [46]).

- Lagerung von angebrochenen Sondenkostflaschen im Kühlschrank; Reste spätestens nach 24 Stunden verwerfen, sofern keine anderen Herstellerangaben vorliegen (Kat. IB). Sondenkost unterliegt der Diätverordnung 2003 (Kat. IV [82]).

- Zubereitung von Tee mit kochendem Wasser (Kat. IB).

- Herstellerangaben bezüglich der Zubereitung der Sondennahrung sind $\mathrm{zu}$ beachten (Kat. IV [46]).

- Schüttelbecher, Messlöffel etc. müssen bei erneutem Gebrauch mit geeigneten Verfahren (z. B. Geschirrspülmaschinen bei $\geq 60^{\circ} \mathrm{C}$ oder in Reinigungs-Desinfektions-Geräten) thermisch desinfizierend aufbereitet werden (Kat. IB).

- Bei transnasaler Sonde tägliche Reinigung von Naseneingang und Sonde (Kat. III).

\subsection{Mundhygiene}

Die Bewohner sollen zur Durchführung einer effektiven Mundhygiene angeleitet und über die Prothesenpflege aufgeklärt werden. Zähne sollten am besten nach jeder Mahlzeit, mindestens aber 2-mal pro Tag, geputzt werden. Auch die Prothesenpflege erfordert besondere Sorgfalt, wobei geeignete bisherige Maßnahmen des Bewohners beibehalten werden können. Sofern die Bewohner nicht in der Lage sind, eine ausreichende Zahn-/Prothesenpflege durchzuführen, ist Hilfestellung zu geben oder die Pflege zu übernehmen. Prothesen regelmäßig auf Plaque und Pilzbe- fall inspizieren und ggf. professionelle Reinigung veranlassen.

\section{Gehäuftes Auftreten \\ von Infektionen \\ (siehe hierzu auch die Empfehlun- gen „Ausbruchsmanagement und strukturiertes Vorgehen bei gehäuftem Auftreten nosokomia- ler Infektionen", ,'Surveillance no- sokomialer Infektionen sowie die Erfassung von Erregern mit speziel- len Resistenzen und Multiresisten- zen" der Kommission für Kranken- haushygiene und Infektionspräven- tion [2])}

Epidemische Häufungen (Ausbrüche) von insbesondere Atemweginfektionen und Infektionen des Magen-Darm-Traktes gehen mit einer hohen Morbidität und Letalität der infektionsanfälligen Bewohner in den Heimen einher [4, 83].

Influenza. Hochkontagiöse Viruserkrankungen der Atemwege sind besonders für ältere Menschen gefährlich. Häufig treten Influenzaausbrüche in Heimen auf, dabei können 25-70\% der Bewohner einer Einrichtung mit zum Teil schweren klinischen Verläufen erkranken, mit einer Letalität von über $10 \%[84,85,86,87]$. Insofern sind Bewohner von Heimen als auch das dort arbeitende Personal eine wichtige Zielgruppe für diejährlich zu wiederholende Grippeschutzimpfung (s. auch Empfehlungen der Ständigen Impfkommission (STIKO)) [88].

Noroviren. Vereinfachte mikrobiologische Nachweisverfahren haben die weite Verbreitung und das epidemische Potenzial von Noroviren deutlich gemacht. Insbesondere in Deutschland, Europa und Nordamerika sind Ausbrüche von Gastroenteritiden durch Noroviren beschrieben worden. Dabei wurde das besondere Risiko von Personen $>69$ Jahren und von Frauen beobachtet. $85 \%$ der Ausbrüche traten in Krankenhäusern sowie Alten- und Pflegeheimen auf [74].

Weitere Erkrankungen bzw. Erreger, die häufig zu Ausbrüchen in Heimen führen, sind in der $\bullet$ Übersicht 1 aufgelistet:

Bei gehäuftem Auftreten von Infektionen ist eine Erfassung und Bewertung (Surveil-
Übersicht 1

Beispiele für Erreger, die mit

Ausbrüchen in Heimen assoziiert

waren

Atemwege

- Influenza [84; 85, 86, 87]

- Andere respiratorische Viren $[89,90]$

Gastrointestinaltrakt

-Virale Gastroenteritis (Noro-, Rotaviren)

[74, 91, 92]

- Salmonellen-Infektionen [93]

-S.-aureus-Lebensmittelintoxikation [93]

Weitere Infektionen

- Keratokonjunctivitis epidemica [94]

- Streptokokken-A-Infektionen [95]

-Scabies-Befall $[70,71]$

lance) der im betreffenden Heim aufgetretenen Infektionen und/oder bestimmter auffallender Erreger (z. B. MRSA, VRE, C. difficile) empfehlenswert, um ggf. die Aktivitäten der Infektionsprävention gezielt ausrichten zu können [96].

\section{Meldepflicht gemäß Infektionsschutzgesetz}

Nach $\$ 6$ Infektionsschutzgesetz (IfSG) sind bestimmte Infektionskrankheiten sowie das gehäufte Auftreten von nosokomialen Infektionen (s. auch Kapitel 7) meldepflichtig. Grundsätzlich ist nach $₫ 8$ IfSG der feststellende Arzt verpflichtet, das Auftreten bzw. den Verdacht der im $\$ 6$ IfSG Abs. (1) und (2) genannten Erkrankungen unverzüglich dem zuständigen Gesundheitsamt namentlich zu melden.

Ist jedoch eine ärztliche Meldung primär nicht erfolgt, muss diese nach $₫ 8$ Abs. (1) Nr. 7 durch den Leiter der Einrichtung bzw. nach $\$ 8$ Abs. (1) Nr. 5 durch einen Angehörigen eines anderen Heil- oder Pflegeberufs (z. B. Krankenschwester, Altenpflegerin) erfolgen. Dies gilt nach $\$ 6$ Abs. (1) Nr. 5 auch beim Auftreten von 2 oder mehr gleichartigen Erkrankungen, wenn ein epidemiologischer Zusammenhang oder eine lebensmittelbedingte Infektionserkrankung anzunehmen ist.

Sofern sich Hinweise auf den Ausbruch einer Infektion ergeben, muss dies gemäß $\$ 6$ Abs. (3) IfSG dem Gesundheitsamt nichtnamentlich gemeldet werden, um den öffentlichen Gesundheitsdienst 
(ÖGD) sowie ggf. einen beratenden Krankenhaushygieniker so früh wie möglich in das Ausbruchmanagement einzubeziehen [40].

\section{Maßnahmen bei Auftreten von Erregern mit besonderen Eigenschaften}

Ein betreuter Wohnbereich, ein Alten-, aber auch ein Pflegeheim stellt den häuslichen Lebensraum für den betroffenen älteren Menschen dar. Daher muss, anders als im Krankenhaus, die Verhältnismäßigkeit zwischen a) einer in Erwägung zu ziehenden Einschränkung der Bewegungsfreiheit und b) dem Schutz der Mitbewohner differenziert und situationsabhängig abgewogen werden. Um dennoch der Verantwortung zur Vermeidung der Weiterverbreitung von Erregern mit besonderen Eigenschaften gerecht zu werden, empfiehlt es sich, auch für Heime für das Auftreten von Erregern, die spezielle Präventionsmaßnahmen erfordern, jeweils detaillierte Vorgehensweisen im Hygieneplan festzulegen.

Bestimmte Grunderkrankungen oder medizinische Maßnahmen können bei einem Bewohner oder Mitbewohner Einfluss auf das Infektionsrisiko haben. So kann z. B. der Kontakt eines an Gürtelrose (Varicella-Zoster-Virus) erkrankten Bewohners dann bei einem anderen Mitbewohner eine generalisierte Zosterinfektion auslösen, wenn dieser zeitgleich immunsupprimiert ist, obgleich er in früheren Jahren eine diesbezügliche Immunkompetenz erworben hatte. Daher ist eine individuelle Risikoeinschätzung für die Weiterverbreitung möglicher Infektionserreger unter Berücksichtigung bestehender Grunderkrankungen oder medizinischer Maßnahmen zusammen mit dem behandelnden Arzt für jeden betroffenen Bewohner bzw. Mitbewohner durchzuführen und mit ggf. erforderlichen Präventionsmaßnahmen zu dokumentieren. Erforderlichenfalls kann der behandelnde Arzt beim zuständigen Gesundheitsamt um Rat bzw. Unterstützung nachsuchen.

Weiter gehende Informationen zu relevanten Erregern bzw. zu übertragbaren Erkrankungen, die in Gemeinschaftseinrichtungen leicht verbreitet werden (z. B. Norovirus-Infektionen, Scabies), können über die Gesundheitsämter sowie Landesoder Bundesgesundheitseinrichtungen eingeholt werden. Die einrichtungsspezifischen Präventionsmaßnahmen sind mit dem beratenden Krankenhaushygieniker oder dem Gesundheitsamt unter Einbeziehung der behandelnden Ärzte schriftlich festzulegen (Hygieneplan).

\subsection{Multiresistente Erreger \\ [z. B. Methicillin-resistente Staphylococcus aureus (MRSA), Vancomycin-resistente Enterokokken (VRE) u. a.]}

Untersuchungen zum Vorkommen von MRSA in Heimen in Deutschland ergaben Prävalenzen zwischen 1,1\% und 2,4\% [20, 21, 97]. In den europäischen Nachbarländern sind sowohl hohe Prävalenzen wie z. B. in Großbritannien bis $17 \%$ und sehr niedrige Prävalenzen unter $1 \%$ wie in den Niederlanden beschrieben [13, 18, 98, 99]. Das Auftreten von MRSA in Heimen ist als Folge des Vorkommens und der Zunahme von MRSA in Krankenhäusern anzusehen, denn es gibt einen engen Zusammenhang zwischen MRSA-Besiedlung von Heimbewohnern und zurückliegenden Krankenhausaufenthalten $[20,100]$. Laut Bradley et al. [101] scheint eine Übertragung von resistenten Stämmen innerhalb von Heimen eher selten zu erfolgen (genotypisch identische Stämme wurden bei Zimmernachbarn in Heimen in den USA bisher selten gefunden). Ausnahmen stellen Ausbruchsituationen dar $[83,102]$.

Jede Institution, die pflegebedürftige Personen betreut (also Krankenhäuser, Rehabilitationskliniken, Heime etc.), muss in der Lage sein, auch solche Menschen zu versorgen, die mit multiresistenten Erregern besiedelt oder infiziert sind. Aufgrund der Tatsache, dass Übertragungen in Einrichtungen der Langzeitpflege und in Altenheimen selten vorkommen und dann gewöhnlich nur zu einer Kolonisierung führen [97, 100], ist eine Ablehnung der Übernahme kolonisierter oder infizierter Personen - sei es in Altenwohnheimen bis hin zu Einrichtungen für Schwerstpflegebedürftige - mit Verweis auf den Nachweis von z. B. MRSA oder gar der Forderung, dass zunächst 3 negative Abstrichergebnisse vorliegen müssen, weder mit organisatorischen noch mit medizinischen $\mathrm{Ar}$ - gumenten und schon gar nicht juristisch $\mathrm{zu}$ rechtfertigen $[4,100,102,103,104]$, denn für die Prävention von Erregerübertragungen - ob MRSA, VRE, ESBL oder andere - ist in der Regel in den hier angesprochenen Einrichtungen die Beachtung der in Kapitel 5 aufgeführten Hygienemaßnahmen angemessen. Dies sind aber genau die Maßnahmen, die ohnehin im Umgang mit jedem Bewohner bzw. Patienten praktiziert werden müssen und zwar unabhängig davon, ob ein auffälliger Erreger festgestellt wurde oder nicht.

Bei den Maßnahmen, die beim Nachweis von multiresistenten Erregern zur Vermeidung ihrer Weiterverbreitung zu ergreifen sind, müssen die Art der Betreuung und Pflege der Bewohner sowie das individuelle Risiko berücksichtigt werden. Die konsequent eingehaltenen StandardHygienemaßnahmen sind als Basis in der Regel ausreichend, um eine Erregerübertragung zu vermeiden. Sie müssen jedoch bei Vorliegen von Risikofaktoren situationsbezogen angepasst, d. h. ggf. ergänzt werden. Deshalb werden im Folgenden spezielle Maßnahmen bei Auftreten multiresistenter Erreger am Beispiel von MRSA zusammenfassend erörtert (s. auch • Tabelle 3). Im Hinblick auf Einschränkungen des Selbstbestimmungsrechtes der Bewohner und therapeutische Optionen können nur solche Maßnahmen empfohlen werden, die aus hygienischer Sicht bewiesenermaßen oder mit hoher Wahrscheinlichkeit das Übertragungsrisiko in Altenund Pflegeheimen reduzieren.

Im Hinblick auf die Vermeidung der Weiterverbreitung sollen alle Mitarbeiter über die in Frage kommenden Erreger, deren Übertragungswege und die notwendigen Maßnahmen bei der Betreuung und Pflege von Personen, die mit speziellen Erregern besiedelt bzw. infiziert sind, ausreichend informiert sein.

\subsubsection{Maßnahmen bei Verlegung und Transport}

Bei Einweisung ehemals oder bekanntermaßen gegenwärtig MRSA-kolonisierter bzw. -infizierter Bewohner und deren unmittelbarer Kontaktpersonen in ein Krankenhaus muss die aufnehmende Einrichtung (der aufnehmende Arzt) entsprechend unterrichtet werden. Das Krankentransportpersonal muss darauf hin- 
gewiesen werden, dass bei engem Direktkontakt mit MRSA-positiven Personen (z. B. beim Umlagern) Einmalhandschuhe und Schutzkittel zu tragen sind. Nach dem Transport sind alle Flächen mit direktem Patientenkontakt (z. B. Krankentransportliege) zu desinfizieren (Wischdesinfektion). Das Begleitpersonal muss eine hygienische Händedesinfektion durchführen. Das Tragen von speziellen Schutzanzügen/Overalls ist beim Transport von MRSA-positiven Personen aus hygienischen Gründen nicht erforderlich und wird in Hinblick auf die von ihnen oder von dieser Schutzkleidung ausgehenden unnötigen und nicht kalkulierbaren Verunsicherung nicht empfohlen.

\subsubsection{Maßnahmen innerhalb der Einrichtung in Abhängigkeit vom individuellen Risiko}

\subsubsection{MRSA-Besiedelung bei} Bewohnern ohne besonderes Risiko Bei MRSA-Besiedelung eines Bewohners ohne chronische Hautläsionen (z. B. Ekzeme, Wunden) und ohne invasive Zugänge (z. B. Harnwegskatheter, PEG-Sonde) unterscheidet sich das Infektionsrisiko für Mitbewohner zunächst nicht von dem in einer häuslichen Lebensgemeinschaft. Vorsichtsmaßnahmen können trotzdem im Einzelfall angebracht sein. So sollen Mitbewohner im gleichen Zimmer keine offenen Wunden haben oder mit Katheter-, Sonden- oder Tracheostoma versorgt sein [97, 98, 100, 105, 106]. Wenn dies nicht möglich ist, ist eine bewohnerbezogene Pflege mit entsprechend zugeordneter Schutzkleidung erforderlich. Außer der Information über den MRSAStatus und ggf. über eine noch nicht abgeschlossene bzw. weiterzuführende Sanierungsbehandlung (siehe unten) bestehen keine Einschränkungen hinsichtlich der Aufnahme in ein Heim.

\subsubsection{MRSA-Besiedelung/Infektion bei Bewohnern mit besonderen Risiken (z. B. invasive Katheter, offene Wunden usw.)}

Einzelzimmer. Einzelzimmer sind nicht generell erforderlich, aber für diese Bewohner in Betracht zu ziehen, wobei allerdings mögliche negative Auswirkungen für die Rehabilitation des betroffenen Bewohners zu berücksichtigen sind. Mitbewohner im selben Zimmer sollten jedoch kein erhöhtes Risiko haben, nach einer eventuellen Besiedelung an MRSA zu erkranken, d. h. keine offenen Wunden haben oder Katheter-, Sonden- oder Tracheostomaträger sein. Ein Zusammenlegen mehrererMRSA-Besiedelter ist möglich [100, 106, 107, 108, 109].

Soziale Kontakte. Soziale Kontakte zu Angehörigen, Besuchern und Mitbewohnern unterliegen keinen Einschränkungen. Besucher müssen keine Schutzkleidung und keine Einmalhandschuhe tragen. Mobile Bewohner können am Gemeinschaftsleben teilnehmen, wenn Hautläsionen/offene Wunden verbunden sind und das Tracheostoma abgedeckt ist. Bei medizinischer Indikation zur Harnableitung muss diese über ein geschlossenes System erfolgen. Besucher und Bewohner sollen zur regelmäßigen Händehygiene angeleitet werden.

Pflegerische Maßnahmen. Pflegerische Maßnahmen müssen im Hinblick auf das Übertragungsrisiko organisiert werden. Sie dürfen bei MRSA-Trägern nicht von Personal mit chronischen Hauterkrankungen durchgeführt werden, da dieses Personal selbst ein erhöhtes Risiko für eine MRSA-Besiedelung hat. Die Pflege muss im Zimmer des Bewohners durchgeführt werden, möglichst nachdem alle anderen Mitbewohner versorgt wurden. Nach pflegerischem Kontakt mit einem MRSA-besiedelten bzw. -infizierten Bewohner müssen die Hände desinfiziert werden. Selbstverständlich muss eine Händedesinfektion auch wie üblich vor und nach pflegerischen Tätigkeiten bei jedem Bewohner mit Wunden, Kathetern und Sonden, außerdem immer auch nach dem Ausziehen von Einmalhandschuhen erfolgen; je nach Tätigkeit müssen Einmalhandschuhe und Schutzkittel bzw. Einmalschürzen beim Umgang mit kontaminierter Bettwäsche, der Versorgung von Blasenkathetern oder beim Verbandswechsel angezogen werden. Ein Mund-Nasen-Schutz ist beim endotrachealen Absaugen unabhängig vom Nachweis von MRSA im Trachealsekret aus generellen Personalschutzgründen (Vermeiden einer Eigenkontamination) empfohlen. Pflegehilfsmittel sind bewohnerbezogen zu verwenden und im Zimmer zu belassen. Vor Anwendung bei anderen Bewohnern müssen sie desinfiziert werden.
Tägliche Reinigung. Die tägliche Reinigung der Oberflächen im Zimmer unterscheidet sich prinzipiell nicht von der in anderen Zimmern, sollte jedoch am Ende eines Durchgangs erfolgen, um eine Weiterverbreitung $\mathrm{zu}$ vermeiden (Information des Reinigungsdienstes). Eine gezielte Desinfektion ist nur bei Kontamination mit Blut, Sekreten oder Exkreten erforderlich. Wäsche, Besteck, Geschirr und Abfälle werden wie üblich behandelt.

- Keine generelle Einzelzimmerunterbringung der Bewohner (Kat. II).

- Unterbringung angepasst an das Risiko (Kat. IB).

\subsubsection{Maßnahmen in Pflegestationen, Schwer-/und Schwerstpflegeeinrichtungen}

In Einrichtungen, in denen die Bewohner überwiegend pflegerisch betreut werden und die Art der medizinischen Versorgung der Bewohner ähnlich der Versorgung von Patienten im Krankenhaus ist, wird als Grundlage für die Festlegung situationsspezifischer Maßnahmen die Berücksichtigung der „Empfehlungen zur Prävention und Kontrolle von Methicillin-resistenten Staphylococcus-aureus- (MRSA-)Stämmen in Krankenhäusern und anderen medizinischen Einrichtungen “ der Kommission für Krankenhaushygiene und Infektionsprävention [2] empfohlen.

\subsection{Mikrobiologische Screeninguntersuchungen}

Routinemäßige Abstrichkontrollen von Bewohnern und Personal in Altenpflegeeinrichtungen werden nicht empfohlen. Ein Bewohner- bzw. Personalscreening kann nach Prüfung der epidemiologischen Gegebenheiten bei gehäuftem Auftreten von MRSA-Infektionen (Ausbruch) notwendig werden, wenn ein Bewohner oder ein Mitglied des Personals als Quelle in Betracht kommt $[4,100,102,110]$.

- Screening von Bewohner und Personal nur bei gehäuftem Auftreten von MRSA-Infektionen (Kat. III).

- Screening bei begründetem Verdacht auf Weiterverbreitung ausgehend von Personal oder Bewohner (Ausbruch) (Kat. IB). 
Tabelle 3

Orientierende Übersicht über Maßnahmen beim Umgang mit MRSA-positiven Bewohnern in Abhängigkeit von der Art und Intensität der Betreuung (s. auch Kapitel 9)

\section{Art der Betreuung Überwiegend sozial \\ Betroffene Personen}

MRSA-positiver Bewohner • Unterbringung:

wie im häuslichen Lebensraum (s. auch 9.1.2)

- Händehygiene:

Händedesinfektion vor Gemeinschaftsaktivitäten

- Sanierung:

nicht routinemäßig; abhängig von der epidemiologi-

schen Situation und dem individuellen Risiko (s. auch 9.3)

Mitbewohner

a) ohne Risikofaktoren

b) mit Risikofaktoren

(s. Tabelle 1)

- Unterbringung: 9.1.2)

- Unterbringung:
In der Regel keine Einschränkungen erforderlich (s. auch

Individuelle Festlegung der Maßnahmen

in Abhängigkeit von der Risikobeurteilung

(s. auch 9.1.2)

• Händehygiene:

Händedesinfektion vor Gemeinschaftsaktivitäten

Personal

• Händehygiene:

Händedesinfektion nach direktem Kontakt mit MRSApositiven Bewohnern und nach dem Ablegen von Einmalhandschuhen

- Einmalhandschuhe:

Bei möglichem Kontakt mit erregerhaltigem Material

- Schutzkleidung:

- Schutzkittel/Schürzen bei engem pflegerischen

Kontakt (z. B. Umbetten)

- Mund-Nasen-Schutz bei möglichem Kontakt mit infektiösen Aerosolen (z. B. bei Tracheostoma-Pflege)

Besucher

Händehygiene:

In der Regel Händewaschen ausreichend

Umgebung

• Flächenreinigung/-desinfektion:
Überwiegend pflegerisch

Zur Vermeidung einer Übertragung sind spezielle Maßnahmen notwendig, bis hin zur Einzelzimmerunterbringung oder Kohortierung (s. hierzu auch 9.1.2)

\section{-Sanierung:}

nicht routinemäßig; abhängig von der epidemiologischen Situation und dem individuellen Risiko (s. auch 9.3)

- Mitbewohner dürfen keinem Übertragungs-

und Infektionsrisiko ausgesetzt sein.

- Besondere Maßnahmen für Mitbewohner können bei offenen Wunden bzw. Hautdefekten, Sonden, Katheter, Tracheostoma erforderlich sein (s. hierzu auch 9.1.2)

\section{• Händehygiene:}

Händedesinfektion nach direktem Bewohnerkontakt, insbesondere vor und nach spezifischen pflegerischen Maßnahmen z. B. Wundversorgung, Harnwegskatheter, PEG-Sonde, Tracheostoma, andere Stomata - vor Verlassen des Zimmers

- nach Ausziehen von Einmalhandschuhen

- Einmalhandschuhe:

Bei möglichem Kontakt mit erregerhaltigem Material

- Schutzkleidung:

- Schutzkittel/Schürzen bei engem pflegerischen Kontakt (z. B. Umbetten)

- Mund-Nasen-Schutz bei möglichem Kontakt mit infektiösen Aerosolen (z. B. bei Tracheostoma-Pflege)

•Händehygiene:

Händedesinfektion vor Verlassen des Zimmers

- Flächenreinigung/-desinfektion:

- nach Reinigungs- und Desinfektionsplan

- gezielte Desinfektion, wenn erforderlich

- Desinfektion von Flächen mit häufigem Hand-/ Hautkontakt ${ }^{a}$

•Wäsche: s. Kapitel 5.3.3

- Betten: s. Tabelle 2 und Kap. 5.3.3

- Geschirr: Übliche (maschinelle) Aufbereitung

Zum Screening siehe 9.2, zur Surveillance siehe 9.4, " Siehe hierzu auch „Anforderungen an die Hygiene bei der Reinigung und Desinfektion von Flächen" Kommissionsempfehlung C.2.1 der Richtlinie für Krankenhaushygiene und Infektionsprävention [2]. 


\subsection{Antiseptische Sanierung von Erregerträgern}

Die Sanierung von MRSA-Trägern ist zwar epidemiologisch wünschenswert, kann jedoch nicht generell gefordert werden. Die Entscheidung für eine Sanierungsbehandlung in einer Altenpflegeeinrichtung sollte in Abhängigkeit von der epidemiologischen Situation und von der individuellen Gefährdung der einzelnen Personen getroffen werden. Der Erfolg der Sanierung wird vom Vorliegen weiterer Risikofaktoren beeinflusst.

Ergebnisse für Sanierungsversuche liegen mit Mupirocin-Nasensalbe und für antiseptische Mundspülung bei Anwendung über 5-7 Tage vor. Antiseptische Körperwaschungen können in Erwägung gezogen werden. Mehrmalige Sanierungsversuche sind nicht sinnvoll. Wenn bereits ein adäquater Sanierungsversuch in einer anderen Einrichtung erfolglos geblieben ist, kann in der Regel auf weitere Versuche verzichtet werden. Mit einem Wiederauftreten von MRSA muss insbesondere bei Bewohnern mit besiedelten chronischen Hautläsionen und dauerhaft invasiven Maßnahmen gerechnet werden [ 97 , 111, 112, 113, 114, 115, 116, 117, 118].

Eine eventuelle Sanierungsbehandlung erfolgt auf ärztliche Anordnung und muss ausreichende Nachuntersuchungen einschließen.

- Sanierungsbehandlung nach Abwägung der Gefährdung des Bewohners und der epidemiologischen Gesamtsituation, keine mehrmaligen Sanierungsversuche (Kat. II).

\subsection{Gehäuftes Auftreten von MRSA-Infektionen}

Ein gehäuftes Auftreten von Infektionen mit MRSA (s. auch Kapitel „Gehäuftes Auftreten von Infektionen und Meldepflicht") muss eine unmittelbare Reaktion der für die Hygiene Verantwortlichen zur Folge haben. Als Erstes müssen epidemiologische Zusammenhänge geklärt und die Hygieneabläufe überprüft werden. Die Meldung an das Gesundheitsamt muss unverzüglich erfolgen ( $\$ 6$ Abs. 3 IfSG). Ggf. ist bei Bewohnern und Personal ein mikrobiologisches Screening erforderlich. Bei anderen multiresistenten Erregern muss in analoger Weise vorgegangen werden.

\section{Weitere Hygienemaßnahmen}

\subsection{Umgang mit Medikamenten und Pflegemitteln \\ (siehe hierzu auch die Empfehlung „Prävention Gefäßkatheter-assozi- ierter Infektionen" der Kommission für Krankenhaushygiene und Infektionsprävention [2])}

Bei betreutem Wohnen ist jeder Heimbewohner für seine eigene Medikation selbst verantwortlich; die Medikamente werden in der betreffenden Wohnung aufbewahrt. Bei zentraler Lagerung und Verabreichung an pflegebedürftige Heimbewohner gelten die gleichen Anforderungen wie in medizinischen Einrichtungen. Medikamente müssen entsprechend den Herstellerangaben gelagert werden. Bei erforderlicher Kühlschranklagerung muss die korrekte Temperatur des Kühlschranks $\left(+2^{\circ} \mathrm{C}-+8^{\circ} \mathrm{C}\right)$ durch regelmäßige Kontrollen sichergestellt werden [119].

Medikamente müssen personenbezogen eingesetzt werden. Das setzt die geeignete Kennzeichnung und Lagerung voraus, sodass eine Verwechslung ausgeschlossen ist. Zu diesem Zweck werden namentlich gekennzeichnete Medikamentenboxen empfohlen. Portionierungsboxen müssen sauber sein (regelmäßige Reinigung z. B. in Geschirrspülmaschinen). Beizentraler Lagerung muss - im Rahmen des heiminternen Qualitätsmanagements - auch eine Überwachung der Verfallsdaten aller Medikamente und aller infektionspräventiv relevanten Hilfsmittel (z. B. steriles Verbandsmaterial) erfolgen. Es wird empfohlen, das heiminterne Vorgehen in der Hygienekommission zu regeln und im Hygieneplan festzuhalten.

- Lagerung von Medikamenten entsprechend Herstellerangaben (Kat. IV [46]).

\subsection{Tierhaltung}

Die Haltung von Haustieren kann für Gesundheit und Wohlbefinden förderlich sein. In Heimen ist das Halten von Tieren grundsätzlich möglich, wenn Unfällen und Infektionsgefährdungen hinreichend vorgebeugt wird und keine Aller- gie bei Mitbewohnern gegen eine Tierhaltung spricht. Die Kriterien für die Aufnahme und Haltung von Tieren sollten von einem Heim schriftlich festgelegt werden. Für weitere Informationen s. auch Gesundheitsberichterstattung des Bundes „Heimtierhaltung - Charncen und Risiken für die Gesundheit“ [120].

Zum Schutz vor Infektionen muss beachtet werden, dass

- das Heimtier ggf. adäquat geimpft und regelmäßig entwurmt wird,

- bei Krankheitszeichen tierärztlich untersucht wird,

- Aufenthaltsbereich, Trink- und Futterbehälter regelmäßig gereinigt,

- Ektoparasiten wie Flöhe, Zecken, Läuse, Milben erkannt und entfernt werden.

\section{Hygienisch-mikrobiologische Überwachung}

(siehe hierzu auch die Empfehlung "Anforderungen an die Hygiene bei der Aufbereitung von Medizinprodukten" der Kommission für Krankenhaushygiene und Infektionsprävention [2])

Entsprechende einrichtungsspezifische Verfahrensvorschriften muss die Heimleitung im Rahmen ihres QM-Programms gemeinsam mit den Hygieneverantwortlichen festlegen. Bei Maßnahmen zur Flächendesinfektion sind keine routinemäßigen Kontrollen (Abklatschuntersuchungen) erforderlich.

\subsection{Maschinelle Aufbereitung von Medizinprodukten}

— Für Geräte zur Aufbereitung von Medizinprodukten (z. B. ReinigungsDesinfektions-Geräte, Sterilisatoren) gelten wie im Krankenhaus die gesetzlichen Vorschriften des Medizinproduktegesetzes [46] und der Medizinprodukte-Betreiberverordnung [47]. Nur für solche Geräte sind die Aufbereitungsverfahren zu validieren und regelmäßig zu überprüfen.

- Für Mehrtankgeschirrspülmaschinen in Gemeinschaftsbereichen wird nach DIN 10510 die einmal jährliche Untersuchung mittels geeigneter Bioindikatoren empfohlen. 
11.2 Dezentrale Desinfektionsmittel-Dosiergeräte (siehe hierzu auch Stellungnahme „Anforderungen an Gestaltung, Eigenschaften und Betrieb von dezentralen DesinfektionsmittelDosiergeräten" Richtlinie der Bundesanstalt für Materialforschung und -prüfung, des Robert KochInstitutes und der Kommission für Krankenhaushygiene und Infektionsprävention [121])

Bei Betrieb von dezentralen Desinfektionsmittel-Dosiergeräten wird empfohlen, die Geräte 1-mal jährlich zu warten [121].

\section{Schutzimpfungen}

Impfungen sind eine der wichtigsten infektionspräventiven Maßnahmen der Medizin und insbesondere in Gemeinschaftseinrichtungen ein wirksames Instrument zur Verhinderung von Ausbrüchen. Dies setzt einen Schutz durch Impfungen bei Bewohner und Personal voraus.

\subsection{Bewohner}

Der aktuelle Impfschutz sollte bei allen Heimbewohnern in Kooperation mit den behandelnden, niedergelassenen Ärzten (s. auch Kapitel 4.4.) unter Zugrundelegung der aktuellen Empfehlungen der STI$\mathrm{KO}[88]$ regelmäßig überprüft werden. $\mathrm{Ne}$ ben einer für alle Erwachsenen empfohlenen Auffrischimpfung gegen Tetanus und Diphtherie sollte eine Influenza-Schutzimpfung jährlich im Herbst jeweils mit der aktuellen von der WHO empfohlenen Antigenkombination für alle Bewohner von Alten- und Pflegeeinrichtungen angeboten werden. Eine Pneumokokken-Schutzimpfung ist bei allen Heimbewohnern über 6o Jahre und bei chronischen Erkrankungen (Wiederholungsimpfung alle 6 Jahre) indiziert.

\subsection{Personal}

Der Arbeitgeber hat gemäß $\$ 15$, $\$ 15$ a und Anhang IV BioStoffV [122] für das Personal vor Aufnahme der Tätigkeit eine arbeitsmedizinische Vorsorgeuntersuchung gegenüber Hepatitis-A- und -B-Virus zu veranlassen und dabei die Impfung an- zubieten, es sei denn, es besteht bei dem Beschäftigten ein ausreichender Immunschutz. Diese Untersuchung ist Voraussetzung für die Tätigkeit. Auch bei anderen tätigkeitsspezifischen Infektionsgefährdungen, z. B. durch Influenza, sind entsprechend der Gefährdungsbeurteilung ggf. eine Impfung anzuraten und zusätzliche Vorsorgeuntersuchungen anzubieten. Die wirksamen in Deutschland zugelassenen Impfstoffe sind in den Impfempfehlungen der STIKO [88] veröffentlicht. Die Beschäftigten sollten darauf hingewiesen werden, regelmäßig den Impfschutz bei von der STIKO für die gesamte Bevölkerung empfohlenen Impfungen gegenüber Diphtherie und Tetanus auffrischen zu lassen.

\section{Lebensmittel- und Küchenhygiene}

Lebensmittel-assoziierteInfektionen undinsbesondere Ausbrüche sind meist Folge von Hygienefehlern bei Kühlung, Zubereitung und Lagerung von Speisen. Die Lebensmittelhygieneverordnung (LMHV) gilt auch für Küchen in Heimen [123]. Es wird in Heimen kein dokumentiertes HACCP-Konzept verlangt, sondern es genügt die Erarbeitung eines Hygieneplans zur Vermeidung der nachteiligen Beeinflussung von Lebensmitteln. Wichtige Voraussetzungen für die Beschäftigung von Personal in der Küche sind:

- die Belehrung durch das Gesundheitsamt gemäß $₫ 43$ IfSG vor Aufnahme der Tätigkeit und

- eine jährliche Wiederholung und Dokumentation der Belehrung durch den Arbeitgeber.

Die Meldepflicht gemäß $₫ 6$ Abs. (2) IfSG muss für den „Verdacht“ auf und die „Erkrankung an" einer mikrobiell bedingten Lebensmittelvergiftung oder an einer akuten infektiösen Gastroenteritis beachtet werden, wenn

- eine Person betroffen ist, die eine Tätigkeit im Sinne des $\$ 42$ Abs. (1) ausübt, oder wenn

- 2 oder mehr gleichartige Erkrankungen auftreten, bei denen ein epidemiologischer Zusammenhang wahrscheinlich ist oder vermutet wird.
Bei Durchfallerkrankungen von Küchenpersonal empfiehlt sich eine bakteriologische und virologische Stuhluntersuchung, um ggf. erregerbezogene Präventionsmaßnahmen einleiten zu können. Über die ordnungsgemäße Lagerung von Lebensmitteln sind die Bewohner, aber auch Besucher bzw. Angehörige entsprechend zu informieren. Einem Befall mit Ungeziefer im Zusammenhang mit Nahrungsmitteln kann am besten durch Sauberkeit und regelmäßige Kontrolle vorgebeugt werden (s. auch Kapitel 5 sowie LMHV). Zur Bekämpfung von Küchenschädlingen muss ggf. (externes) Fachpersonal beauftragt werden.

- Erarbeitung eines Hygieneplans für den Küchenbetrieb (Kat. IV [123]).

- Belehrung durch das Gesundheitsamt gemäß $₫ 43$ IfSG vor Aufnahme der Tätigkeit (Kat. IV [40]).

— Jährliche Wiederholung der Belehrung durch den Arbeitgeber mit entsprechender Dokumentation(Kat. IV [40]).

- Meldepflicht gemäß $\$ 6$ Abs. (2) IfSG bei Verdacht und Erkrankung an einer mikrobiell bedingten Lebensmittelvergiftung oder an einer akuten infektiösen Gastroenteritis für Küchenpersonal (Kat. IV [40]).

- Bei Durchfall bakteriologische und virologische Stuhluntersuchung bei Küchenpersonal (Kat. IB).

\subsection{Essgeschirr}

Wie im Haushalt kann Geschirr in Eintankgeschirrspülautomaten (Haushaltsgeschirrspülmaschine mit $65^{\circ} \mathrm{C}$-Programm) gereinigt werden. Bei großem Geschirraufkommen kann eine Mehrtankgeschirrspülmaschine sinnvoll sein.

\section{Abfallentsorgung}

Der in Heimen anfallende Abfall ist nicht gewerblich und unterliegt daher der kommunalen Abfallsatzung. Aus diesem Grund sollte ein einrichtungsspezifisches Konzept in Form eines Entsorgungsplanes in Anlehnung an den Vorgaben des LAGA-Merkblatts und der kommunalen Regelungen erstellt werden [124]. 


\section{Anforderungen an externe Dienstleister}

Mit externen Dienstleistern (z. B. Reinigungsunternehmen, Wäschereien, Küche, Maniküre, Pediküre, Friseure) muss vertraglich geregelt werden, dass sich diese Firmen an die von der Einrichtung im Hygieneplan festgelegten Hygienemaßnahmen halten müssen. Die Aufstellung eigener Regeln muss kompatibel zur Institution sein, um ein für die Institution einheitliches Infektionspräventionskonzept umsetzen zu können.

\section{Bauliche Anforderungen und Hygienemaßnahmen bei Umbau und Rekonstruktion}

Gebäude, Räume und Ausstattungen müssen der Heimmindestbauverordnung [128], den baurechtlichen Anforderungen im jeweiligen Bundesland, den berufsgenossenschaftlichen Vorschriften [129], den Bestimmungen der Arbeitsstättenverordnung [130], den brandschutztechnischen Vorschriften sowie den Normen zur barrierefreien und körperbehindertengerechten Gestaltung (DIN 19024 und 18025) genügen. In Altenpflegeeinrichtungen können Baumaßnahmen insbesondere für Personen mit Immunsuppression durch Freisetzung von Schimmelpilzsporen eine relevante Infektionsgefährdung darstellen. Dadurch mitbedingte Anpassung an ihre Unterbringung wie auch ihre Behandlung müssen mit dem behandelnden Arzt und ggf. einem beratenden Krankenhaushygieniker abgestimmt werden.

\section{Anhang 1: Beispielhafte Aufstellung von Themen und Inhalten eines Hygiene- plans, modifiziert nach P. Bergen [131].}

- Hygieneorganisation/Hygienemanagement

- Personelle Organisation

- Hygienebeauftragte

- Qualitätszirkel „Infektionsprävention“

- Personalhygiene

- Händehygiene

- Schutzkleidung

- Infektionsschutz am Arbeitsplatz

- Hygiene bei speziellen medizinischpflegerischen Maßnahmen

- Umgang mit Arzneimitteln
- Aufbereitung von Medizinprodukten und Pflegeartikeln

- Flächenreinigung und -desinfektion

- Reinigungs- und Desinfektionspläne

- Verhalten im Infektionsfall

- Meldepflicht

- Maßnahmen beim Auftreten von Erregern mit besonderen Eigenschaften

- Ausbruchsmanagement

- Umgang mit Verstorbenen

- Küchenhygiene

- Personalhygiene

- Eigenkontrollkonzept

- Wäscheversorgung und -entsorgung

- Umgebungshygiene

- Wartung und Überprüfunghaustechnischer Einrichtungen und Geräte

Wasserhygiene

- Abfall

- Abfallplan

- Sonstiges

- Schädlingsbekämpfung

- Heimtierhaltung

Die Empfehlungen wurden ehrenamtlich und ohne Einflussnahme kommerzieller Interessengruppen im Auftrag der Kommission für Krankenhaushygiene und Infektionsprävention bearbeitet von: H.M. Just (Nürnberg, Leiter der Arbeitsgruppe), M.-E. Höpken (Hannover), I. Kappstein (München), E. Kunoth (München), I. Daniels-Haardt (Münster), A. Kramer (Greifswald), M. Exner (Bonn), G. Unger (Bad Elster), N. Wischnewski (Berlin), M. Mielke (Berlin) und von den Mitgliedern der Kommission für Krankenhaushygiene und Infektionsprävention genehmigt.

\section{Literatur}

1. RückertW (2001) Prävention, Rehabilitation und Tagespflege sollten ausgebaut werden Sonst werden Jahr für Jahr über 10.000 zusätzliche Heimplätze benötigt. Pro Alter - Kuratorium Deutsche Altershilfe 1:37-39

2. Robert Koch-Institut (Hrsg) (2004) Richtlinie für Krankenhaushygiene und Infektionsprävention. Elsevier Verlag, Lose Blattsammlung, 1. Auflage bzw. http://www.rki.de > Infektionsschutz > Krankenhaushygiene $>$ Kommission für Krankenhaushygiene und Infektionsprävention

3. Pitten FA, Rosin M, Kramer A (2001) Leitlinienentwurf: Indikationen und Wirkstoffauswahl zur prophylaktischen und therapeutischen Mundhöhlenantiseptik. Hyg Med 10:418-424

4. Smith PW, Rusnak PG (1997) SHEA/APIC position paper: infection prevention and control in the long-term facility. Infect Control Hosp Epidemiol 18:831-849

5. Nicolle LE (2001) Extended care facilities and nursing homes. In: Abrutyn E, Goldman DA, Scheckler WE (eds) Infection control reference service the experts' guide to the guidelines, 2nd edn.W.B. Saunders Company, Philadelphia, pp 95-138
6. Garibaldi RA (1999) Residential care and the elderly: the burden of infection. J Hosp Infect 43 [Suppl]:S9-S18

7. Strausbaugh LJ, Joseph CL (2000) The burden of infection in long-term care. Infect Control Hosp Epidemiol 21:674-679

8. Nicolle LE (2000) Infection control in long-term care facilities. Clin Infect Dis 31:752-756

9. Golliot F, Astagneau P, Cassou B et al. (2001) Nosocomial infections in geriatric long-term care and rehabilitation facilities: exploration in the development of a risk index for epidemiological surveillance. Infect Control Hosp Epidemiol 22:746-753

10. Marrie TJ (2002) Pneumonia in the long-term care facility. Infect Control Hosp Epidemiol 23:159-164

11. Strausbaugh LJ, Joseph CL (1999) Epidemiology and prevention of infections in residents of longterm care facilities. In: Mayhall GC (ed) Hospital epidemiology and infection control. Lippincott Williams \& Wilkins, Philadelphia, pp 1461-1482

12. Nurse BA, Garibaldi RA (1998) Infections in long term care facilities. In: Bennett JV, Brachman PS (eds) Hospital infections, 4th edn. Lippincott-Raven, Philadelphia, pp 689-724

13. Stone SP (1999) Soil, seed and climate: developing a strategy for prevention and management of infections in UK nursing homes. J Hosp Infect 43 [Suppl]:29-38

13a. Engelhart ST, Hanses-Derendorf L, Exner M, Kramer MH (2005) Prospective surveillance for healthcare-associated infections in German nursing home residents. J Hosp Infect 60:46-50

14. Mott PD, Barker WH (1988) Treatment decisions for infections occuring in nursing home-residents. J Am Geriatr Soc 36:820-824

15. Kerr HD, Byrd JC (1991) Nursing home patients transferred by ambulance to a VA emergency department. J Am Geriatr Soc 39:132-139

16. Heuck D, Nassauer A (1999) Methicillin-resistente Staphylococcus aureus in Alten- und Pflegeheimen. Hyg Med 3:72-80

17. Trick WE, Weinstein RA, DeMarais PL et al. (2001) Colonization of skilled-care facility residents with antimicrobial-resistant pathogens. J Am Geriatr Soc 49:270-276

18. Strausbaugh LJ, Crossley KB, Nurse BA et al. (1996) SHEA position paper: antimicrobial resistance in long-term care facilities. Infect Control Hosp Epidemiol 17:129-140

19. Bradley SF (2002) Staphylococcus aureus infections and antibiotic resistance in older adults. Clin Infect Dis 34:211-216

20. Heuck D, Fell G, Hamouda O et al. (2000) Erste Ergebnisse einer überregionalen Studie zur MRSABesiedlung bei Bewohnern von Alten- und Pflegeheimen. Hyg Med 25:191-192

21. Heudorf U, BremerV, Heuck D (2001) MRSA-Besiedelung bei Bewohnern von Alten- und Pflegeheimen sowie bei Patienten einer geriatrischen Rehabilitationsklinik in Frankfurt am Main, 1999. Gesundheitswesen 63:447-454

22. Braak $N$ van den, Ott $A$, Belkum $A$ van et al. (2000) Prevalence and determinants of fecal colonization with vancomycin-resistant Enterococcus in hospitalized patients in The Netherlands. Infect Control Hosp Epidemiol 21:520-524

23. Neuhaus B, Bocter N, Braulke C et al. (2002) Studie zum Vorkommen von Methicillin-resistenten Staphylococcus aureus in Alten- und Pflegeheimen. Bundesgesundheitsblatt 45:894-904

24. McCue JD (1999) Antibiotic use in the elderly: issues and nonissues. Clin Infect Dis 28:750-752 
25. Höpken ME, Dreesman J, Braulke C et al. (2001) MRSA-Besiedlung in einem Alten- und Pflegeheim: Risikofaktoren und Prävalenz. Hyg Med 26:225-230

26. Drittes Gesetz zur Änderung des Heimgesetzes vom 5. November 2001. Bundesgesetzblatt 2001, Teil I Nr. 57 vom 09.11.2001, S 2960-2980

27. SENIC: Haley RW, Culver DH, White JW (1985) The efficacy of infection surveillance and control programs in preventing nosocomial infections in US hospitals. Am J Epidemiol 121:182-205

28. Rüden H, Daschner F, Gastmeier P (Hrsg) (2000) Krankenhausinfektionen - Empfehlungen für das Hygienemanagement. Springer Verlag, Berlin Heidelberg New York Tokyo

29. Kappstein I (Hrsg.) (2004) Nosokomiale Infektionen - Prävention, Labor-Diagnostik, Antimikrobielle Therapie, 3. Aufl. W. Zuckschwerdt Verlag, München

30. Kramer A, Heeg P, Botzenhart K (Hrsg) (2001) Krankenhaus- und Praxishygiene. Urban \& Fischer, München

31. Makris AT, Morgan L, Gaber DJ et al. (2000) Effect of a comprehensive infection control program on the incidence of infections in long-term care facilities. Am J Infect Control 28(1):3-7

32. Daly PB, Smith PW, Rusnak PG et al. (1992) Impact on knowledge and practice of a multiregional long-term care facility infection control training program. Am J Infect Control 20(5):225-233

33. Smith PW (1998) Infection control in long-term care facilities. Infect Dis Clin Pract 7:91-95

34. Verordnung über personelle Anforderungen für Heime (Heimpersonalverordnung) (1993) Bundesgesetzblatt I 1205

35. Bundesgesundheitsministerium für Familie, Senioren, Frauen und Jugend: Bekanntmachung der Neufassung des Altenpflegegesetzes vom 25. August 2003, Bundesgesetzblatt 2003 Teil I Nr. 44, ausgegeben zu Bonn am 4. September 2003

36. Bundesgesundheitsministerium für Familie, Senioren, Frauen und Jugend: Ausbildungs- und Prüfverordnung für den Beruf der Altenpflegerin und des Altenpflegers (Altenpflege-Ausbildungs- und Prüfungsverordnung-AltPflAPrV) vom 26. November 2002, Bundesgesetzblatt 2002, Teil I Nr. 81, ausgegeben zu Bonn am 29. November 2002

37. Sektion „Hygiene in der ambulanten und stationären Kranken- und Altenpflege/Rehabilitation" der Deutschen Gesellschaft für Krankenhaushygiene (DGKH) (2002) Leitlinie: Hygienebeauftragte(r) in Pflegeeinrichtungen. Hyg Med 6:271-272

38. Loeb M (2000) Antibiotic use in long-term care facilities: many unanswered questions. Infect Control Hosp Epidemiol 21:680-683

39. Mylotte JM (1999) Antimicrobial prescribing in long-term care facilities: prospective evaluation of potential antimicrobial use and cost indicators. Am J Infect Control 27:10-19

40. Gesetz zur Neuordnung seuchenrechtlicher Vorschriften (Seuchenrechtsneuordnungsgesetz SeuchRNeuG) (Infektionsschutzgesetz). Bundesgesetzblatt Jahrgang 2000 Teil I Nr. 33, ausgegeben zu Bonn am 25. Juli 2000; 1045-1077

41. Technische Regel für Biologische Arbeitsstoffe (TRBA) 250: Biologische Arbeitsstoffe im Gesundheitswesen und in der Wohlfahrtspflege. Bundesarbeitsblatt 11/2003; 53-73 http://www.baua. de/prax/in

42. Bales S, Baumann HG (2003) § 36 Einhaltung der Infektionshygiene. In: Bales S, Baumann HG, Schnitzler N (Hrsg) Infektionsschutzgesetz Kommentar und Vorschriftensammlung, 2. Aufl. Kohlhammer, Stuttgart, S 247-249
43. Sozialgesetzbuch V Sozialgesetzbuch - Fünftes Buch (V) - Gesetzliche Krankenversicherung (Artikel 1 des Gesetzes v. 20. Dezember 1988), BGBI I, S 2477-2482

44. Puzniak LA, Leet T, Mayfield J et al. (2002) To gown or not to gown: the effect on acquisition of vancomycin-resistant enterococci. Clin Infect Dis (United States) 35(1):18-25

45. Srinivasan A, Song X, Ross T et al. (2002) A prospective study to determine whether cover gowns in addition to gloves decrease nosocomial transmission of vancomycin-resistant enterococci in an intensive care unit. Infect Control Hosp Epidemiol (United States) 23(8):424-428

46. Medizinproduktegesetz, BGBI I 1994, 1963; neu gefasst i. d. Bek. v. 7.8.2002 I 3146; geändert durch Art. 109 v. 25.11 .2003 I 2304

47. Medizinprodukte-Betreiber-Verordnung, BGBL I, 2001, S 3854; geändert durch Art. 1 § 10 am 4.12.2002, BGBL I S 4456; geändert am 14.2.2004 BGBL IS 216

48. Takahashi A, Yomoda S, Tanimoto K et al. (1998) Streptococcus pyogenes hospital-acquired infection within a dermatological ward. J Hosp Infect (England) 40(2):135-140

49. Shiomori T, Miyamoto H, Makishima Ket al. (2002) Evaluation of bedmaking-related airborne and surface methicillin-resistant Staphylococcus aureus contamination. J Hosp Infect (England) 50(1):30-35

49a. Berufsgenossenschaftliche Regeln für Sicherheit und Gesundheit bei der Arbeit (BGR) 500 (2004) Berufsgenossenschaft für Gesundheitsdienst und Wohlfahrtspflege-BGW (Hrsg), Stand 12/2004, Druckhaus Dresden

50. Falkiner FR (1993) The insertion and management of indwelling urethral catheters - minimizing the risk of infection. J Hosp Infect 25:79-90

51. Nicolle LE, SHEA Long-Term-Care-Committee (2001) Urinary tract infections in long-term-carefacilities. Infect Control Hosp Epidemiol 22:167-175

52. Piechota H, Brühl P, Hertle L, Sökeland J (2000) Katheterdrainage der Harnblase heute. Dtsch Ärztebl 97:S168-174

53. Stickler DJ, Chawla JC (1987) The role of antiseptics in the management of patients with longterm indwelling bladder catheters. J Hosp Infect 10:219-228

54. Warren JW(1997) Urinary tract infections. In: Wenzel RP (ed) Prevention and control of nosocomial infections, 3rd edn. Williams \& Wilkins, Baltimore, pp $821-840$

55. Wyndaele JJ (2002) Intermittent catheterization: which is the optimal technique? Spinal Cord 40(9):432-437

56. Shekelle PG, Morton SC, Clark KA et al. (1999) Sys tematic review of risk factors for urinary tract infection in adults with spinal cord dysfunction. J Spinal Cord Med 22(4):258-272

57. Feil H (Hrsg) (2001) Stomapflege/Enterostomatherapie: Stoma- und Wundversorgung, 7. Aufl. Schlütersche Verlagsanstalt, Hannover

58. Peters-GawlikM (Hrsg) (1998) Praxishandbuch Stomapflege: Beratung, Betreuung und Versorgung Betroffener, 1. Aufl. Ullstein Medical, Wiesbaden

59. Ravenschlag C, Piechota HJ (im Druck) Stomatherapie. In: Fenger $\mathrm{H}$, Kolb GF, Raem AM et al. (Hrsg) Handbuch Geriatrie und Gerontologie - Leitfaden für Praktiker und Kliniker, 1. Aufl. Deutsche Krankenhaus-Verlagsgesellschaft (DKVG), Düsseldorf

60. O' Grady NP, Alexander M, Dellinger EP et al. (2002) Guidelines for the prevention of intravascular catheter-related infections. Infect Control Hosp Epidemiol (United States) 23(12):759-69
61. Stout JE, Brennen C, Muder RR (2000) Legionnaires' disease in a newly constructed long-term care facility. J Am Geriatr Soc 48:1589-1592

62. Maesaki S, Kohno S, Koga H et al. (1992) An outbreak of legionnaires' pneumonia in a nursing home. Intern Med 31:508-512

63. Yu VL (2000) Nosocomial legionellosis. Curr Opin Infect Dis 13:385-388

64. Robert Koch-Institut (2004) Zum Welttuberkulosetag: Situation in Europa und Deutschland. Epidemiol Bull 12:95-97

65. Yoshikawa TT (1994) The challenge and unique aspects of tuberculosis in older patients. Infect Dis Clin Pract 3:62-66

66. Castle SC (2000) Clinical relevance of age-related immune dysfunction. Clin Infect Dis 31:578-585

67. Tablan OC, Anderson LJ, Besser R et al. (2004) Guidelines for Preventing Health-Care- Associated Pneumonia, 2003. Recommendations of CDC and the Healthcare Infection Control Practices Advisory Committee. MMWR 53(RR03):1-36

68. Mangram AJ, Horan TC, Pearson ML et al. (1999) Guideline for prevention of surgical site infection, 1999. Infect Control Hosp Epidemiol 20:247-280

69. Schiemann D (Hrsg) (2000) Expertenstandard Decubitusprophylaxe in der Pflege. Deutsches Netzwerk für Qualitätssicherung in der Pflege, Fachhochschule Osnabrück

70. Robert Koch-Institut (1998) Scabies-Ausbruch in einem Altenheim: Erfahrungsbericht. Epidemiol Bull 35:249-251

71. Robert Koch-Institut (1997) Scabies in Heimen. Epidemiol Bull 21:143-145

72. Chrintz H, Vibits H, Cordtz TO et al. (1989) Need for surgical wound dressing. Br J Surg 76:204-205

73. Winkler R (Hrsg) (1993) Stomapflege und Versorgung. In: Stomatherapie. Atlas und Leitfaden für intestinale Stomata, 3. Aufl. Georg Thieme Verlag, Stuttgart New York, S 21-34

74. Robert Koch-Institut (2003) Erkrankungen durch Norwalk-ähnliche Viren (Noroviren). Epidemiol Bull 6:39-41

75. Bennet RG (1993) Diarrhea among residents of long-term care facilities. Infect Control Hosp Epidemiol 14:397-404

76. Nicholson FB, Korman MG, Richardson MA (2000) Percutaneous endoscopic gastrostomy: a review of indications, complications and outcome. J Gastroenterol Hepatol 15:21-25

77. American Gastroenterological Association (1995) Technical review on tube feeding for enteral nutrition. Gastroenterology 108:1282-1301

78. American Society for Gastrointestinal Endoscopy (1998) Role of PEG/PEJ in enteral feeding. Gastrointest Endosc 48:699-701

79. French Society of Digestive Endoscopy (1999) Guidelines of the French Society of Digestive Endoscopy (SFED): endoscopic gastrostomy. Endoscopy 31:207-208

80. Schura G, Löser C (2001) Pflegerichtlinien für die Langzeitbetreuung von Patienten mit enteralen Sondensystemen. In: Löser C, Keymling M (Hrsg) Praxis der enteralen Ernährung, 1. Aufl. Georg Thieme Verlag, Stuttgart, S 221-233

81. Beattie TK, Anderton A (1999) Microbiological evaluation of four enteral feeding systems which have been deliberately subjected to faulty handling procedures. J Hosp Infect 42:11-20

82. 12. Verordnung zur Änderung der Diätverordnung 2003, BGBI 2003, Teil I, Nr. 13 ausgegeben zu Bonn am 8. April 2003; 467-476 (Diätverordnung BGBI I 1963,415) 
83. Nicolle LE, Strausbaugh LJ, Garibaldi RA (1996) Infections and antibiotic resistance in nursing homes. Clin Microbiol Rev 9(1):1-17

84. Goodman RA, Orenstein WA, Munro TF et al. (1982) Impact of influenza A in a nursing home. JAMA 247:1451-1453

85. Gravenstein S, Miller BA, Drink P (1992) Prevention and control of influenza $A$ outbreaks in longterm care facilities. Infect Control Hosp Epidemiol 13:49-54

86. Horman JT, Stetler HC, Israel E et al. (1986) An outbreak of influenza $A$ in a nursing home. Am J Public Health 76:501-503

87. Staynor K, Forster G, Mc Atlier M et al. (1994) Influenza $A$ outbreak in a nursing home: the value of early diagnosis and the use of amantadine hydrochloride. Can J Infect Control 9:109-112

88. Ständige Impfkommission (STIKO) (2005) Empfehlungen der ständigen Impfkommission Stand Juli 2005. Epidemiol Bull 30:257-272

89. Falsley AR (1991) Noninfluenza respiratory virus infection in long-term care facilities. Infect Control Hosp Epidemiol 12:602-608

90. Agius G, Dindinaud G, Biggar RJ et al. (1990) An epidemic of respiratory syncytial virus in elderly people. J Med Virol 30:117-127

91. Marrie T, Lee S, Faultzner R et al. (1982) Rotavirus infection in a geriatric population. Arch Intern Med 142:313-316

92. Pegues D, Woernle I (1993) An outbreak of acute nonbacterial gastroenteritis in a nursing home. Infect Control Hosp Epidemiol 14:87-94

93. Levine WC, Smart JF, Archer D et al. (1991) Foodborn disease outbreak in nursing homes, 1975 through 1987. JAMA 266:2105-2109

94. Boustacha E, Nicolle LE (1995) Conjunctivitis in a long-term care facility. Infect Control Hosp Epidemiol 16:210-216

95. Schwartz B, Ussery XT (1992) Group A Streptococcal outbreaks in nursing homes. Infect Control Hosp Epidemiol 13:742-747

96. Ammon A, Gastmeier P, Weist K et al. (2001) Empfehlungen zur Untersuchung von Ausbrüchen nosokomialer Infektionen. In: Robert Koch-Institut (Hrsg) Heft 21. Robert-Koch Institut, Berlin

97. Bock-Hensley O, Baum H von, Schmidt $\mathrm{C}$ et al. (2002) MRSA Management in Alten- und Pflegeheimen. Hyg Med 27:11-15

98. Boyce JM (1992) Methicillin-resistant Staphylococcus aureus in hospitals and long-term care facilities: microbiology, epidemiology, and preventive measures. Infect Control Hosp Epidemiol 13:725737

99. Dutch Workingparty Infection Prevention (2003) Policy for Methicillin-resistant Staphylococcus aureus, 2003, 1-20, http://www.wip.nl

100. Bradley SF (1997) Methicillin-resistant Staphylococcus aureus in nursing homes - epidemiology, prevention and management. Drug Aging 10:185-198

101. Bradley SF (1999) Issues in the management of resistant bacteria in long-term care facilities. Infect Contr Hosp Epidemiol 20:362-366

102. Mylotte JM (1994) Control of methicillin-resistant Staphylococcus aureus: the ambivalence persists. Infect Control Hosp Epidemiol 15:73-77

103. Duckworth G, Heathcock R (1995) Guidelines on the control of methicillin-resistant Staphylococcus aureus in the community. Report of a combined Working Party of the British Society for Antimicrobial Chemotherapy and the Hospital Infection Society. J Hosp Infect 31:1-12
104. Boyce JM, Jackson MM, Pugliese G et al. (1994) Methicillin-resistant Staphylococcus aureus (MRSA): a briefing for acute care hospitals and nursing facilities. Infect Control Hosp Epidemiol 15:105-115

105. Bradley SF (1994) MRSA in long-term care: fact, fiction, and controversy. Infect Dis Clin Pract 3:321-326

106. Thomas JC, Bridge J, Waterman S et al. (1989) Transmission and control of methicillin-resistant Staphylococcus aureus in a skilled nursing facility. Infect Control Hosp Epidemiol 10:106-110

107. O'Sullican NP, Keane CT (2000) Risk factors for colonization with methicillin-resistant Staphylococcus aureus among nursing home residents. J Hosp Infect 45:206-210

108. Muder RR, Brenner C, Wagener MM et al. (1991) Methicillin-resistant Staphylococcal colonization and infection in a long-term care facility. Ann Int Med 114(2):107-112

109. Larson E, Bobo L, Bennett R et al. (1991) Lack of care giver hand contamination with endemic bacterial pathogens in a nursing home. Am J Infect Control 19:11-15

110. Lessing MPA, Jordens JZ, Bowler ICJ (1996) When should healthcare workers be screened for methicillin-resistant Staphylococcus aureus? J Hosp Infect 34:205-210

111. Dietlein E, Hornei B, Krizek L et al. (2002) Empfehlungen zur Kontrolle von MRSA in Alten- und Pflegeheimen sowie Rehabilitationskliniken - ein Diskussionsbeitrag. Hyg Med 27:131-137

112. Kauffman CA, Bradley SF, Terpenning MS et al. (1993) Attemps to eradicate methicillin-resistant Staphylococcus aureus from a long-term care facility with the use of mupirocin ointment. Am J Med 94:371-378

113. Harbarth S, Liassine N, Dharan S et al. (2000) Risk factors for persistent carriage of methicillinresistant Staphylococcus aureus. Clin Infect Dis 31:1380-1385

114. Scanvic A, Denic L, Gaillon S et al. (2001) Duration of colonization by methicillin-resistant Staphylococcus aureus after hospital discharge and risk factors for prolonged carriage. Clin Infect Dis 32:1393-1398

115. Harbarth S, Dharan S, Liassine N et al. (1999) Randomized, placebo-controlled, double-blind trial to evaluate the efficacy of mupirocin for eradicating carriage of methicillin-resistant Staphylococcus aureus. Antimicrob Agents Chemother 43:1412-1416

116. MacKinnon MM, Allen KD (2000) Long-term MRSA carriage in hospital patients. J Hosp Infect 46:216-221

117. Blok HE, Vriens MR et al. (2001) Carriage of methicillin-resistant Staphylococcus aureus (MRSA) after discharge from hospital: follow-up for how long? A Dutch multi-centre study. J Hosp Infect 48:325-327

118. Watanakunakorn C, Axelson C, Bota B et al. (1995) Mupirocin ointment with and without chlorhexidin baths in the eradication of Staphylococcus aureus nasal carriage in nursing home residents. Am J Infect Control 23:306-309

119. Europäisches Arzneibuch, 4. Ausgabe, Grundwerk 2002 (Ph. Eur. 4.00) und Nachträge, Deutscher Apotheker Verlag, Stuttgart

120. Gesundheitsberichterstattung des Bundes (2003) Heimtierhaltung - Chancen und Risiken für die Gesundheit. Robert Koch-Institut, Heft 19
121. Bundesanstalt für Materialforschung und -prüfung und Kommission für Krankenhaushygiene und Infektionsprävention (2004) Anforderungen an Gestaltung, Eigenschaften und Betrieb von dezentralen Desinfektionsmittel-Dosiergeräten, Richtlinie der Bundesanstalt für Materialforschung und -prüfung und der Kommission für Krankenhaushygiene und Infektionsprävention. Bundesgesundheitsblatt 47:67-72

122. Biostoffverordnung BGBI I 1999, 50; ausgegeben zu Bonn am 27. Januar 1999, zuletzt geändert durch Art. 8V v. 23.12.2004 I 3758

123. Lebensmittelhygiene-Verordnung (Artikel 1 der Verordnung über Lebensmittelhygiene und zur Änderung der LebensmitteltransportbehälterVerordnung) (LMHV), BGBL I 1997, S 2008

124. Richtlinie über die ordnungsgemäße Entsorgung von Abfällen aus Einrichtungen des Gesundheitsdienstes (2002) In: Robert Koch- Institut (Hrsg) Richtlinie für Krankenhaushygiene und Infektionsprävention, Elsevier Verlag, 2004, Lose Blattsammlung, 1. Auflage München

125. Crossley K, SHEA Long-Term-Care Committee (1998) SHEA position paper:Vancomycin-resistant enterococci in long-term-care facilities. Infect Control Hosp Epidemiol 19:521-525

126. Safdar N, Maki DG (2002) The commonality of risk factors for nosocomial colonization and infection with antimicrobial-resistant Staphylococcus aureus, enterococcus, gram-negative bacilli, Clostridium difficile and Candida. Ann Intern Med 136:834-844

127. Sandoval C, Walter SD, McGeer A et al. (2004) Nursing home residents and Enterobacteriaceae resistant to third-generation cephalosporins. Emerg Infect Dis 10:1050-1055

128. Verordnung über bauliche Mindestanforderungen für Altenheime, Altenwohnheime und Pflegeheime für Volljährige. BGBI I 1978, 189, Stand: Änderung durch Art. 5 V v. 25.11.2003 I 2346

129. BGV1 Grundsätze der Prävention. Berufsgenossenschaftliche Vorschriften für Sicherheit und Gesundheit bei der Arbeit. Unfallverhütungsvorschriften BG-Vorschriften, Stand: Oktober 2002

130. Verordnung über Arbeitsstätten (2004) Bundesgesetzblatt Jahrgang 2004 Teil I, Nr. 44, ausgegeben zu Bonn am 24. August 2004. S 2179-2189

131. Bergen $P(2004)$ Hygieneplaninhalte. In: Bergen $P$ (Hrsg) Hygiene in Altenpflegeheimen. Urban \& Fischer Verlag, München, S 180-183 